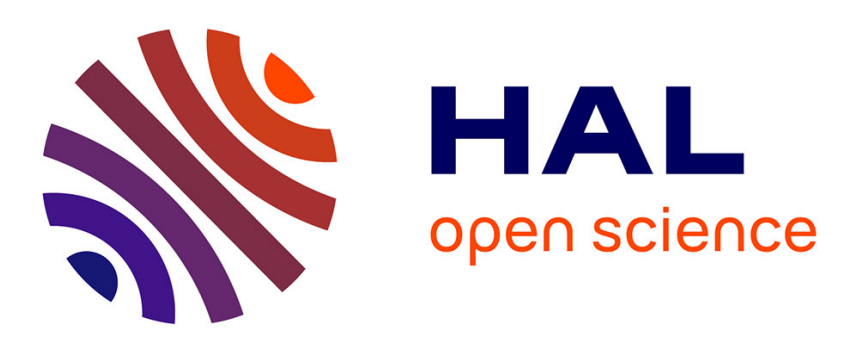

\title{
Properties, performance and practical interest of the widely linear MMSE beamformer for nonrectilinear signals
}

Pascal Chevalier, Jean-Pierre Delmas, Abdelkader Oukaci

\section{- To cite this version:}

Pascal Chevalier, Jean-Pierre Delmas, Abdelkader Oukaci. Properties, performance and practical interest of the widely linear MMSE beamformer for nonrectilinear signals. Signal Processing, 2014, 97, pp.269 - 281. 10.1016/j.sigpro.2013.09.017 . hal-01285813

\section{HAL Id: hal-01285813 \\ https://hal.science/hal-01285813}

Submitted on 9 Mar 2016

HAL is a multi-disciplinary open access archive for the deposit and dissemination of scientific research documents, whether they are published or not. The documents may come from teaching and research institutions in France or abroad, or from public or private research centers.
L'archive ouverte pluridisciplinaire HAL, est destinée au dépôt et à la diffusion de documents scientifiques de niveau recherche, publiés ou non, émanant des établissements d'enseignement et de recherche français ou étrangers, des laboratoires publics ou privés. 


\title{
Properties, performance and practical interest of
}

\section{the widely linear MMSE beamformer for}

\section{nonrectilinear signals}

\author{
Pascal Chevalier, Jean-Pierre Delmas and Abdelkader Oukaci
}

\begin{abstract}
Widely Linear (WL) Minimum Mean Square Error (MMSE) estimation has received a great interest these last twenty years for second order (SO) noncircular signals. In the context of radio communications networks, it has been shown in particular that WL MMSE receivers allow to implement Single Antenna Interference Cancellation (SAIC) of one rectilinear interference, such as Binary Phase Shift Keying (BPSK) or Amplitude Shift Keying (ASK) interference, or of quasi-rectilinear interference, such as Minimum Shift keying (MSK), Gaussian MSK (GMSK) or Offset Quadrature Amplitude Modulation (OQAM) interference, hence their great interest for Global System for Mobile Communications (GSM) cellular networks in particular. However, one may wonder whether WL MMSE receivers remain attractive for SO noncircular nonrectilinear interferences, not so scarce in practice. The purpose of this paper is mainly to answer to this important question by giving, in a self-contained and unified way, some new insights into the behaviour, properties and performance of the WL MMSE beamformer in the presence of arbitrary noncircular signals and interference which are not necessarily rectilinear. It is shown in particular that, surprisingly, WL MMSE receivers lose their practical interest for strong interferences which are not rectilinear. This breakthrough thus generates a new open problem for the choice between linear and WL MMSE receiver corresponding to the detection of rectilinearity (and/or quasirectilinearity), instead of noncircularity, in a given noisy observation. Although this question is out of the scope of this paper, we finally propose preliminary tools based on blind source separation methods to solve this problem.
\end{abstract}

\section{Index Terms}

Noncircular, Widely linear, (non)Rectilinear, MMSE, Interferences, SAIC, Beamformers, Improper, Rectilinearity Detection, Blind Source Separation.

Pascal Chevalier is with CNAM, CEDRIC laboratory, 292 rue St Martin, 75141 Paris Cedex, France and with Thales-Communications, AMS/TCP, 4 rue des Louvresses, 92622 Gennevilliers Cedex, France, e-mail: pascal.chevalier@cnam.fr, phone: +(33).1.46.13.26.98, fax: $+(33) \cdot 1 \cdot 46.13 \cdot 25.55$.

Jean-Pierre Delmas and Abdelkader Oukaci are with Institut TELECOM, TELECOM SudParis, Département CITI, CNRS UMR 5157, 91011 Evry Cedex, France, e-mail: jean-pierre.delmas(abdelkader.oukaci)@it-sudparis.eu, phone: +(33).1.60.76.46.32, fax: $+(33) \cdot 1 \cdot 60.76 .44 .33$. 
Paper accepted to Signal Processing.

\section{INTRODUCTION}

WL MMSE estimation have received a great interest these last twenty years for SO noncircular (or improper) [27] signals [28], [7], [11], [12], [13], [6], [21], [25], [30]. In the context of radio communications networks using rectilinear modulations such as BPSK or ASK modulations [29], it has been shown in particular in [7], [11], [12], [13], [21] that the WL MMSE receiver allows to implement SAIC of one intra-network interference in contrast to linear MMSE receiver. This SAIC concept can also be implemented for radio communication networks using quasi-rectilinear modulations, i.e., modulations whose complex amplitude can be considered, after a derotation operation, as a filtered version of a rectilinear modulation, such as MSK, GMSK or OQAM modulations [29], hence its great interest for GSM networks in particular [31], [26].

However, one may wonder whether WL MMSE receivers remain attractive for SO noncircular interferences which are not rectilinear, nevertheless not so scarce in practice. Such signals may correspond, for example, to frequency bins of speech signals as discussed recently in [1], [2] or to rectangular QAM modulations [29], which may present a potential interest in multi-user contexts associated with WL receivers, similarly to the avantages of ASK over QAM modulations presented in [24], [21]. An other example of nonrectilinear noncircular signal seen by the receiver appears after the SO statistics estimation, over a finite interval duration, of a rectilinear or a quasi-rectilinear (after a derotation) signal having a non zero frequency offset [8].

The scarce papers dealing with WL receivers in the presence of noncircular nonrectilinear signals correspond to [1], [2] for noise reduction of speech signals and to [22], [23], [9], [10] for radiocommunications. Papers [22], [23] limit the analysis to a specific case corresponding to mixtures of Pulse Amplitude Modulated (PAM), i.e., rectilinear, and QAM, i.e. SO circular, signals. Papers [9], [10], written by the authors, are conference papers associated with the present paper which introduce sparsely some materials of the paper. Moreover the absence of guiding line in [9], [10] to enlighten the breakthrough between rectilinear and noncircular nonrectilinear strong interferences may prevent the reader of these papers to catch the main message.

In this context, the purpose of this paper is mainly to give, in a self-contained, coherent, unified, guided and progressive way, some new insights, some of which are borrowed from the conference papers [9], [10], into the behaviour, properties and performance, in terms of output Signal to Interference plus Noise Ratio (SINR) and Symbol Error Rate (SER), of the WL MMSE beamfomer in order to evaluate its practical interest for noncircular signals and interferences which are not rectilinear. It is proved in particular that, surprisingly, WL MMSE receivers lose their practical interest for strong interferences which are not rectilinear. This breakthrough thus generates a new open problem for the choice between linear and WL MMSE receiver corresponding to the detection of rectilinearity (or quasi-rectilinearity), instead of noncircularity, in a given noisy observation. Although this question is out of the scope of this paper, we finally propose preliminary tools based on blind 
source separation methods to solve this problem.

This paper is organized as follows. After the introduction of some hypotheses and data statistics in section II, section III recalls the WL MMSE beamformer and presents some of its properties and performance in arbitrary SO noncircular context. A detailed analysis of its performance and practical interest for noncircular nonrectilinear signal and/or interferences is presented in section IV. Section V describes preliminary tools for rectilinearity (or quasi-rectilinearity) blind detection. Finally section VI provides a discussion and some concluding remarks.

\section{HyPOTHESES AND DATA STATISTICS}

\section{A. Hypotheses}

We consider an array of $N$ narrow-band (NB) sensors and we denote by $\mathbf{x}(t)$ the vector of complex amplitudes of the signals at the output of these sensors. Each sensor is assumed to receive the contribution of a signal of interest (SOI) corrupted by a total noise (potentially composed of interferences and background noise). Under these assumptions, the observation vector $\mathbf{x}(t)$ can be written as follows

$$
\mathbf{x}(t)=s(t) \mathbf{s}+\mathbf{n}(t)
$$

where $s(t)$ corresponds, to within a potential frequency offset, to the complex amplitude of the SOI, assumed to be zero mean and potentially SO noncircular, $\mathrm{s}$ is the steering or the channel vector of the SOI (whose first entry is constrained to be one) and $\mathbf{n}(t)$ is the total noise vector assumed to be potentially SO noncircular and statistically uncorrelated with the SOI $s(t)$. Note that model (1) seems to assume propagation channels with no delay spread, which occurs, for example, for free space propagation (spectrum monitoring from plane, unmanned aerial vehicle or satellite) or flat fading channels (spectrum monitoring in some urban radio communications situations). However, it may also take into account propagation channels with delay spread for which uncorrelated multipaths are processed as particular interfering sources.

In order to introduce WL filtering in the following, we define the extended observation vector $\widetilde{\mathbf{x}}(t) \stackrel{\text { def }}{=}$ $\left[\mathbf{x}^{T}(t), \mathbf{x}^{H}(t)\right]^{T}$, where $T$ and $H$ means transpose and transpose and conjugate, respectively. Using (1) we obtain:

$$
\widetilde{\mathbf{x}}(t)=s(t) \widetilde{\mathbf{s}}_{1}+s^{*}(t) \widetilde{\mathbf{s}}_{2}+\widetilde{\mathbf{n}}(t)=\mathbf{S} \widetilde{\mathbf{s}}(t)+\widetilde{\mathbf{n}}(t),
$$

where $\widetilde{\mathbf{n}}(t) \stackrel{\text { def }}{=}\left[\mathbf{n}^{T}(t), \mathbf{n}^{H}(t)\right]^{T}, \widetilde{\mathbf{s}}_{1} \stackrel{\text { def }}{=}\left[\mathbf{s}^{T}, \mathbf{0}_{N}^{T}\right]^{T}, \widetilde{\mathbf{s}}_{2} \stackrel{\text { def }}{=}\left[\mathbf{0}_{N}^{T}, \mathbf{s}^{H}\right]^{T}, \mathbf{0}_{N}$ is the $N \times 1$ null vector, $\mathbf{S} \stackrel{\text { def }}{=}\left[\widetilde{\mathbf{s}}_{1}, \widetilde{\mathbf{s}}_{2}\right]$ and $\widetilde{\mathbf{s}}(t) \stackrel{\text { def }}{=}\left[s(t), s^{*}(t)\right]^{T}$. 


\section{B. SO statistics}

The SO statistics (SOS) of $\mathbf{x}(t)$ which are exploited in the following, correspond to matrices $\mathbf{R}_{x}$ and $\mathbf{C}_{x}$ defined by

$$
\begin{aligned}
& \mathbf{R}_{x} \stackrel{\text { def }}{=}<\mathrm{E}\left[\mathbf{x}(t) \mathbf{x}^{H}(t)\right]>\stackrel{\text { def }}{=} \pi_{s} \mathbf{s s}^{H}+\mathbf{R}_{n}, \\
& \mathbf{C}_{x} \stackrel{\text { def }}{=}<\mathrm{E}\left[\mathbf{x}(t) \mathbf{x}^{T}(t)\right]>\stackrel{\text { def }}{=} \pi_{s} \gamma_{s} \mathbf{s s}^{T}+\mathbf{C}_{n},
\end{aligned}
$$

where $<$. $>$ denotes the time averaging operation ${ }^{1}$, with respect to $t$, over the window $\left[-T_{0} / 2, T_{0} / 2\right], \pi_{s} \stackrel{\text { def }}{=}<$ $\mathrm{E}\left[|s(t)|^{2}\right]>$ is the time averaged power of the SOI received by the first sensor, $\gamma_{s} \stackrel{\text { def }}{=}<\mathrm{E}\left[s(t)^{2}\right]>/ \pi_{s} \stackrel{\text { def }}{=}$ $\left|\gamma_{s}\right| e^{2 i \phi_{s}}$ such that $0 \leq\left|\gamma_{s}\right| \leq 1$, is the time averaged SO noncircularity coefficient of the SOI seen by the receiver, $\mathbf{R}_{n} \stackrel{\text { def }}{=}<\mathrm{E}\left[\mathbf{n}(t) \mathbf{n}^{H}(t)\right]>$ and $\mathbf{C}_{n} \stackrel{\text { def }}{=}<\mathrm{E}\left[\mathbf{n}(t) \mathbf{n}^{T}(t)\right]>$ are respectively the time averaged correlation and complementary correlation matrices of the total noise, respectively. The receiver will see a SO noncircular total noise $\mathbf{n}(t)$ (resp. SOI $s(t)$ ) only if the matrix $\mathbf{C}_{n}$ (resp., $\gamma_{s}$ ) is not equal to zero. The SOI is seen as rectilinear (resp., SO circular) if and only if $\left|\gamma_{s}\right|=1$ (resp. $\gamma_{s}=0$ ), whereas it is seen as SO noncircular and nonrectilinear if $\left(0<\left|\gamma_{s}\right|<1\right)$.

The SOS of $\widetilde{\mathbf{x}}(t)$ which are exploited in the following correspond to the matrix $\mathbf{R}_{\tilde{x}}$ defined by

$$
\mathbf{R}_{\tilde{x}} \stackrel{\text { def }}{=}<\mathrm{E}\left[\widetilde{\mathbf{x}}(t) \widetilde{\mathbf{x}}^{H}(t)\right]>\stackrel{\text { def }}{=} \mathbf{S R}_{\tilde{s}} \mathbf{S}^{H}+\mathbf{R}_{\tilde{n}}
$$

where $\mathbf{R}_{\tilde{s}} \stackrel{\text { def }}{=}<\mathrm{E}\left[\widetilde{\mathbf{s}}(t) \widetilde{\mathbf{s}}^{H}(t)\right]>$ and where the matrix $\mathbf{R}_{\tilde{n}} \stackrel{\text { def }}{=}<\mathrm{E}\left[\widetilde{\mathbf{n}}(t) \widetilde{\mathbf{n}}^{H}(t)\right]>$ can be written as

$$
\mathbf{R}_{\tilde{n}}=\left[\begin{array}{cc}
\mathbf{R}_{n} & \mathbf{C}_{n} \\
\mathbf{C}_{n}^{*} & \mathbf{R}_{n}^{*}
\end{array}\right] .
$$

III. THE WIDELY LINEAR MMSE BEAMFORMER

\section{A. Presentation and Adaptive Implementation}

The WL MMSE beamformer [28], [11], corresponds to the WL filter $\widetilde{\mathbf{w}}$ whose output $y(t) \stackrel{\text { def }}{=} \widetilde{\mathbf{w}}^{H} \widetilde{\mathbf{x}}(t)$ minimizes the time-averaged MSE criterion defined by

$$
\operatorname{MSE}[\widetilde{\mathbf{w}}]=<\mathrm{E}\left[\left|s(t)-\widetilde{\mathbf{w}}^{H} \widetilde{\mathbf{x}}(t)\right|^{2}\right]>
$$

It is straightforward to show that the vector $\widetilde{\mathbf{w}}$ minimizing (7) is defined by

$$
\widetilde{\mathbf{w}}_{\mathrm{MMSE}} \stackrel{\text { def }}{=} \mathbf{R}_{\tilde{x}}^{-1} \mathbf{r}_{\tilde{x}, s}
$$

where $\mathbf{r}_{\tilde{x}, s} \stackrel{\text { def }}{=}<\mathrm{E}\left[\widetilde{\mathbf{x}}(t) s^{*}(t)\right]>$. Note that when $(\mathbf{x}(t), s(t))$ are jointly SO circular, $\widetilde{\mathbf{w}}_{\text {MMSE }}$ reduces to the linear MMSE beamformer [28], $\mathbf{w}_{\text {MMSE }} \stackrel{\text { def }}{=} \mathbf{R}_{x}^{-1} \mathbf{r}_{x, s}$ with $\mathbf{r}_{x, s} \stackrel{\text { def }}{=}<\mathrm{E}\left[\mathbf{x}(t) s^{*}(t)\right]>$. In practical situations,

\footnotetext{
${ }^{1}$ Note that the signals are not necessarily SO stationary.
} 
$\mathbf{R}_{\tilde{x}}$ and $\mathbf{r}_{\tilde{x}, s}$ are not known a priori and have to be estimated from a training sequence using a Least Square estimation approach [11].

\section{B. Enlightening interpretation}

We give in this section, for SOI with arbitrary noncircularity property, an enlightening interpretation of the WL MMSE beamformer, initially introduced in [9], allowing to understand its better behavior with respect to both the Capon's beamformer [3], [4] and the WL Minimum Variance Distorsionless Response (MVDR) beamformer introduced in [8]. To this aim, let us note that for a SOI which is seen as SO noncircular, i.e. such that $\gamma_{s} \neq 0, s^{*}(t)$ is correlated with $s(t)$ and contains both a SOI and an interference component. To compute the SOI component of $s^{*}(t)$, let us consider the Hilbert space of random processes having a finite time-averaged power and fitted with the inner product $(u(t), v(t)) \stackrel{\text { def }}{=}<\mathrm{E}\left[u(t) v^{*}(t)\right]>$. It is then easy to compute the orthogonal projection of $s^{*}(t)$ onto $s(t)$ for the previous inner product. It is straightforward to show [8] that $s^{*}(t)$ can be written as

$$
s^{*}(t)=\gamma_{s}^{*} s(t)+\left[\pi_{s}\left(1-\left|\gamma_{s}^{2}\right|\right]^{1 / 2} i_{s}(t),\right.
$$

where $<\mathrm{E}\left[s(t) i_{s}^{*}(t)\right]>=0$ and $<\mathrm{E}\left[\left|i_{s}(t)\right|^{2}\right]>=1$. It is also easy to verify from straightforward manipulations of (9) that $<\mathrm{E}\left[s(t) i_{s}(t)\right]>=\left[\pi_{s}\left(1-\left|\gamma_{s}\right|^{2}\right)\right]^{1 / 2}$ and $<\mathrm{E}\left[i_{s}^{2}(t)\right]>=-\gamma_{s}^{*}$. Expression (9) shows that, for a given time-averaged useful input power $\pi_{s}$, the time-averaged power of the SOI component of $s^{*}(t)$ is equal to $\pi_{s}\left|\gamma_{s}\right|^{2}$ and increases with $\left|\gamma_{s}\right|$. In particular for a rectilinear SOI $\left(\left|\gamma_{s}\right|=1\right), s^{*}(t)=e^{-2 i \phi_{s}} s(t)$ and $s^{*}(t)$ totally corresponds to the SOI, whereas for a SO circular SOI $\left(\gamma_{s}=0\right), s^{*}(t)=\pi_{s}^{1 / 2} i_{s}(t)$ and $s^{*}(t)$ totally corresponds to an interference for the SOI. Using (9) into (2) we obtain

$$
\widetilde{\mathbf{x}}(t)=s(t)\left[\widetilde{\mathbf{s}}_{1}+\gamma_{s}^{*} \widetilde{\mathbf{s}}_{2}\right]+\left[\pi_{s}\left(1-\left|\gamma_{s}^{2}\right|\right]^{1 / 2} i_{s}(t) \widetilde{\mathbf{s}}_{2}+\widetilde{\mathbf{n}}(t) \stackrel{\text { def }}{=} s(t) \widetilde{\mathbf{s}}_{\gamma}+\widetilde{\mathbf{n}}_{\gamma}(t)\right.
$$

where $\widetilde{\mathbf{s}}_{\gamma} \stackrel{\text { def }}{=} \widetilde{\mathbf{s}}_{1}+\gamma_{s}^{*} \widetilde{\mathbf{s}}_{2}=\left[\mathbf{s}^{T}, \gamma_{s}^{*} \mathbf{s}^{H}\right]^{T}$ and $\widetilde{\mathbf{n}}_{\gamma}(t) \stackrel{\text { def }}{=}\left[\pi_{s}\left(1-\left|\gamma_{s}^{2}\right|^{1 / 2}\right] i_{s}(t) \widetilde{\mathbf{s}}_{2}+\widetilde{\mathbf{n}}(t)\right.$ are respectively the equivalent extended steering vector of the SOI and the global noise component, uncorrelated with $s(t)$, for the extended observation vector $\widetilde{\mathbf{x}}(t)$. Using (10) into (8), we finally obtain

$$
\widetilde{\mathbf{w}}_{\mathrm{MMSE}}=\pi_{s} \mathbf{R}_{\tilde{x}}^{-1} \widetilde{\mathbf{S}}_{\gamma}=\frac{\pi_{s}}{1+\pi_{s} \widetilde{\mathbf{s}}_{\gamma}^{H} \mathbf{R}_{\tilde{n}_{\gamma}}^{-1} \widetilde{\mathbf{s}}_{\gamma}} \mathbf{R}_{\tilde{n}_{\gamma}}^{-1} \widetilde{\mathbf{s}}_{\gamma}
$$

where $\mathbf{R}_{\tilde{n}_{\gamma}} \stackrel{\text { def }}{=}<\mathrm{E}\left[\widetilde{\mathbf{n}}_{\gamma}(t) \widetilde{\mathbf{n}}_{\gamma}^{H}(t)\right]>$ is the time-averaged correlation matrix of $\widetilde{\mathbf{n}}_{\gamma}(t)$. Denoting by $\widetilde{\mathbf{w}}_{\mathrm{MVDR}_{2}}$ the WL MVDR beamformer that minimizes the time-averaged output power $\widetilde{\mathbf{w}}^{H} \mathbf{R}_{\tilde{x}} \widetilde{\mathbf{w}}$ under the constraint $\widetilde{\mathbf{w}}^{H} \widetilde{\mathbf{s}}_{\gamma}=1$, it is straightforward to verify that $\widetilde{\mathbf{w}}_{\mathrm{MVDR}_{2}}$ is defined by

$$
\widetilde{\mathbf{w}}_{\mathrm{MVDR}_{2}} \stackrel{\text { def }}{=}\left[\widetilde{\mathbf{s}}_{\gamma}^{H} \mathbf{R}_{\tilde{x}}^{-1} \widetilde{\mathbf{s}}_{\gamma}\right]^{-1} \mathbf{R}_{\tilde{x}}^{-1} \widetilde{\mathbf{s}}_{\gamma}=\left[\widetilde{\mathbf{s}}_{\gamma}^{H} \mathbf{R}_{\tilde{n}_{\gamma}}^{-1} \widetilde{\mathbf{s}}_{\gamma}\right] \mathbf{R}_{\tilde{n}_{\gamma}}^{-1} \widetilde{\mathbf{s}}_{\gamma}
$$


We then deduce from (11) and (12) that similarly to the linear MMSE beamformer which is colinear to the MVDR beamformer, the WL MMSE beamformer $\widetilde{\mathbf{w}}_{\mathrm{MMSE}}$ is colinear to the WL MVDR beamformer $\widetilde{\mathbf{w}}_{\mathrm{MVDR}_{2}}$ and we obtain:

$$
\widetilde{\mathbf{w}}_{\mathrm{MMSE}}=\frac{\pi_{s} \widetilde{\mathbf{s}}_{\gamma}^{H} \mathbf{R}_{\tilde{n}_{\gamma}}^{-1} \widetilde{\mathbf{s}}_{\gamma}}{1+\pi_{s} \widetilde{\mathbf{s}}_{\gamma}^{H} \mathbf{R}_{\tilde{n}_{\gamma}}^{-1} \widetilde{\mathbf{s}}_{\gamma}} \widetilde{\mathbf{w}}_{\mathrm{MVDR}_{2}} .
$$

Note that the MVDR beamformer $\widetilde{\mathbf{w}}_{\mathrm{MVDR}_{2}}$ depends on both $\mathbf{s}$ and $\gamma_{s}$ and keeps the whole SOI component contained in $\tilde{\mathbf{x}}(t)$ contrary to the WL MVDR beamformer introduced in [8], which minimizes $\widetilde{\mathbf{w}}^{H} \mathbf{R}_{\tilde{x}} \widetilde{\mathbf{w}}$ under the constraint $\widetilde{\mathbf{w}}^{H} \widetilde{\mathbf{s}}_{1}=1$ and $\widetilde{\mathbf{w}}^{H} \widetilde{\mathbf{S}}_{2}=0$ and which is defined by

$$
\widetilde{\mathbf{w}}_{\mathrm{MVDR}_{1}} \stackrel{\text { def }}{=} \mathbf{R}_{\tilde{x}}^{-1} \mathbf{S}\left[\mathbf{S}^{H} \mathbf{R}_{\tilde{x}}^{-1} \mathbf{S}\right]^{-1} \mathbf{f}=\mathbf{R}_{\tilde{n}}^{-1} \mathbf{S}\left[\mathbf{S}^{H} \mathbf{R}_{\tilde{n}}^{-1} \mathbf{S}\right]^{-1} \mathbf{f},
$$

where $\mathbf{f} \stackrel{\text { def }}{=}[1,0]^{T}$. This beamformer does not depend on $\gamma_{s}$ and nulls completely the $s^{*}(t)$ part of the SOI component of $\widetilde{\mathbf{x}}(t)$. Moreover, while the implementation of $\widetilde{\mathbf{w}}_{\mathrm{MVDR}_{1}}$ requires the knowledge or the estimation of $\mathbf{s}$ and $\mathbf{R}_{\tilde{x}}$, that of $\widetilde{\mathbf{w}}_{\mathrm{MVDR}_{2}}$ requires the knowledge or estimation of both $\gamma_{s}, \mathbf{s}$ and $\mathbf{R}_{\tilde{x}}$ or, to within a scalar, the use of a training sequence which is correlated with $s(t)$ and not correlated with the total noise. Finally, note that both $\widetilde{\mathbf{w}}_{\mathrm{MVDR}_{1}}$ and $\widetilde{\mathbf{w}}_{\mathrm{MVDR}_{2}}$ fit the well-known Capon's beamformer [3], [4], defined by

$$
\mathbf{w}_{\mathrm{CAPON}} \stackrel{\text { def }}{=}\left[\mathbf{s}^{H} \mathbf{R}_{x}^{-1} \mathbf{s}\right]^{-1} \mathbf{R}_{x}^{-1} \mathbf{s}=\left[\mathbf{s}^{H} \mathbf{R}_{n}^{-1} \mathbf{s}\right]^{-1} \mathbf{R}_{n}^{-1} \mathbf{s}
$$

when respectively the total noise is SO circular $\left(\mathbf{C}_{n}=\mathbf{0}\right)$ and both the SOI and the total noise are SO circular $\left(\gamma_{s}=0\right.$ and $\left.\mathbf{C}_{n}=\mathbf{0}\right)$.

\section{WL GSC Structure}

It can be easily verified that the WL beamformer $\widetilde{\mathbf{w}}_{\mathrm{MVDR}_{2}}$ has an equivalent WL Generalized Sidelobe Canceller (GSC) structure [17] depicted on Figure 1, where $\widetilde{\mathbf{w}}_{f}$ is an $2 N \times 1$ deterministic WL spatial filter such that $\widetilde{\mathbf{w}}_{f}^{H} \widetilde{\mathbf{s}}_{\gamma}=1$ and its output is given by $y_{f}(t) \stackrel{\text { def }}{=} \widetilde{\mathbf{w}}_{f}^{H} \widetilde{\mathbf{x}}(t) . \mathbf{F}$ is a full-rank $(2 N-1) \times 2 N$ blocking matrix verifying $\mathbf{F} \widetilde{s}_{\gamma}=\mathbf{0}_{2 N-1}$ and whose output corresponds to the $(2 N-1) \times 1$ vector $\widetilde{\mathbf{z}}(t) \stackrel{\text { def }}{=} \mathbf{F} \widetilde{\mathbf{x}}(t), \widetilde{\mathbf{w}}_{a}$ is the $(2 N-1) \times 1$ WL spatial filter which generates the output $y_{a}(t) \stackrel{\text { def }}{=} \widetilde{\mathbf{w}}_{a}^{H} \widetilde{\mathbf{z}}(t)$ and which minimizes the timeaveraged power of the output $y(t) \stackrel{\text { def }}{=} y_{f}(t)-y_{a}(t)$. More precisely, under the previous assumptions, it can be verified that the equivalent $2 N \times 1$ WL spatial filter, $\widetilde{\mathbf{w}}_{\mathrm{GSC}}$, which generates the same output, $y(t) \stackrel{\text { def }}{=} \widetilde{\mathbf{w}}_{\mathrm{GSC}}^{H} \widetilde{\mathbf{x}}(t)$, from the extended observation vector $\widetilde{\mathbf{x}}(t)$ is defined by (12). We finally remark that for both SO circular SOI $\left(\gamma_{s}=0\right)$ and total noise $\left(\mathbf{C}_{n}=\mathbf{0}\right)$, the WL GSC structure is the linear functional scheme depicted in [17].

\section{General SINR Performance}

1) General SINR computation and link with MSE criterion:

From (10) it is easy to compute the ratio of the time-averaged powers of the SOI component and the associated 
global noise component at the output of an arbitrary TI WL filter $\widetilde{\mathbf{w}}$, referred to as the SINR at the output of $\widetilde{\mathbf{w}}$, defined by

$$
\operatorname{SINR}[\widetilde{\mathbf{w}}]=\pi_{s} \frac{\left|\widetilde{\mathbf{w}}^{H} \widetilde{\mathbf{s}}_{\gamma}\right|^{2}}{\widetilde{\mathbf{w}}^{H} \mathbf{R}_{\tilde{n}_{\gamma}} \widetilde{\mathbf{w}}}
$$

It is easy to verify that the WL filters which maximize this output SINR are collinear to $\widetilde{\mathbf{w}}_{\mathrm{MMSE}}$ and $\widetilde{\mathbf{w}}_{\mathrm{MVDR}}$ Using (11) to (15) into (16), we obtain the SINR at the output of the previous beamformers given respectively by:

$$
\begin{aligned}
\operatorname{SINR}_{\mathrm{MMSE}} & \stackrel{\text { def }}{=} \operatorname{SINR}\left[\widetilde{\mathbf{w}}_{\mathrm{MMSE}}\right]=\operatorname{SINR}_{\mathrm{MVDR}_{2}} \stackrel{\text { def }}{=} \operatorname{SINR}\left[\widetilde{\mathbf{w}}_{\mathrm{MVDR}_{2}}\right]=\pi_{s} \widetilde{\mathbf{s}}_{\gamma}^{H} \mathbf{R}_{\tilde{n}_{\gamma}}^{-1} \widetilde{\mathbf{s}}_{\gamma}, \\
\mathrm{SINR}_{\mathrm{MVDR}_{1}} & \stackrel{\text { def }}{=} \operatorname{SINR}\left[\widetilde{\mathbf{w}}_{\mathrm{MVDR}_{1}}\right]=\frac{\pi_{s}}{\mathbf{f}^{H}\left[\mathbf{S}^{H} \mathbf{R}_{\tilde{n}}^{-1} \mathbf{S}\right]^{-1} \mathbf{f}}, \\
\operatorname{SINR}_{\mathrm{CAPON}} & \stackrel{\text { def }}{=} \operatorname{SINR}\left[\left(\mathbf{w}_{\mathrm{CAPON}}^{T}, \mathbf{0}^{T}\right)^{T}\right]=\pi_{s} \mathbf{s}^{H} \mathbf{R}_{n}^{-1} \mathbf{s} .
\end{aligned}
$$

Using the fact that $\mathbf{w}_{\mathrm{CAPON}}, \widetilde{\mathbf{w}}_{\mathrm{MVDR}_{1}}$ and $\widetilde{\mathbf{w}}_{\mathrm{MVDR}_{2}}$ minimize the output power $\widetilde{\mathbf{w}}^{H} \mathbf{R}_{\tilde{x}} \widetilde{\mathbf{w}}$ under different constraints that are included, the inclusion principle allows us to prove that generally

$$
\mathrm{SINR}_{\mathrm{CAPON}} \leq \mathrm{SINR}_{\mathrm{MVDR}_{1}} \leq \mathrm{SINR}_{\mathrm{MVDR}_{2}}=\mathrm{SINR}_{\mathrm{MMSE}}
$$

Moreover, inserting (10) into (7) and using (16), we obtain the general relation

$$
\operatorname{MSE}[\widetilde{\mathbf{w}}]=\pi_{s}\left(\left|1-\widetilde{\mathbf{w}}^{H} \widetilde{\mathbf{s}}_{\gamma}\right|^{2}+\frac{\left|\widetilde{\mathbf{w}}^{H} \widetilde{\mathbf{s}}_{\gamma}\right|^{2}}{\operatorname{SINR}[\widetilde{\mathbf{w}}]}\right)
$$

which shows in particular that the WL filter $\widetilde{\mathbf{w}}$ which minimizes MSE[ $\widetilde{\mathbf{w}}]$ under the constraint $\widetilde{\mathbf{w}} \widetilde{\mathbf{s}}_{\gamma}=1$ is also the WL filter $\widetilde{\mathbf{w}}$ which maximizes $\operatorname{SINR}[\widetilde{\mathbf{w}}]$ under the same constraint, which corresponds to $\widetilde{\mathbf{w}}_{\mathrm{MVDR}_{2}}$. This shows that under the constraint $\widetilde{\mathbf{w}}^{H} \widetilde{\mathbf{s}}_{\gamma}=1$, SINR maximization and MSE minimization are equivalent criteria, which gives a physical interpretation of the SINR criterion (16) in terms of MSE minimization. Without the constraint $\widetilde{\mathbf{w}}^{H} \widetilde{\mathbf{S}}_{\gamma}=1$, (21) shows that MSE minimization is no longer equivalent to SINR maximization, but the WL filter, $\widetilde{\mathbf{w}}_{\text {MMSE}}$, which minimizes MSE $[\widetilde{\mathbf{w}}]$ also maximizes $\operatorname{SINR}[\widetilde{\mathbf{w}}]$ but is not the only one. From the previous results, we obtain

$$
\operatorname{MSE}\left[\widetilde{\mathbf{w}}_{\mathrm{MVDR}_{2}}\right]=\frac{\pi_{s}}{\operatorname{SINR}_{\mathrm{MMSE}}}=\frac{1}{\widetilde{\mathbf{s}}_{\gamma}^{H} \mathbf{R}_{\tilde{n}_{\gamma}}^{-} \widetilde{\mathbf{s}}_{\gamma}}
$$

which is greater than MSE[ $\left.\widetilde{\mathbf{w}}_{\mathrm{MMSE}}\right]$ defined by

$$
\operatorname{MSE}\left[\widetilde{\mathbf{w}}_{\mathrm{MMSE}}\right]=\pi_{s}-\mathbf{r}_{\tilde{x}, s}^{H} \mathbf{R}_{\tilde{x}}^{-1} \mathbf{r}_{\tilde{x}, s}=\frac{\pi_{s}}{1+\pi_{s} \widetilde{\mathbf{s}}_{\gamma}^{H} \mathbf{R}_{\tilde{n}_{\gamma}}^{-1} \widetilde{\mathbf{s}}_{\gamma}}=\frac{\pi_{s}}{1+\operatorname{SINR}_{\mathrm{MMSE}}},
$$

and which tends toward MSE $\left[\widetilde{\mathbf{w}}_{\mathrm{MMSE}}\right]$ as $\operatorname{SINR}_{\mathrm{MMSE}} \gg 1$.

2) SINR computation as a function of $\left|\gamma_{s}\right|$ :

To evaluate the impact, on the performance, of the SO non circularity coefficient $\gamma_{s}$ of the SOI, it is necessary 
to compute $\operatorname{SINR}_{\text {MMSE }}$ as a function of $\left|\gamma_{s}\right|$ for an arbitrary total noise. From (6), we derive that $\mathbf{R}_{\tilde{n}}^{-1}$ can be written as

$$
\mathbf{R}_{\tilde{n}}^{-1}=\left[\begin{array}{cc}
\mathbf{A} & \mathbf{D} \\
\mathbf{D}^{*} & \mathbf{A}^{*}
\end{array}\right],
$$

where the $N \times N$ Hermitian matrix A and complex symmetric matrix $\mathbf{D}$ are given by [11]

$$
\begin{aligned}
& \mathbf{A} \stackrel{\text { def }}{=}\left[\mathbf{R}_{n}-\mathbf{C}_{n} \mathbf{R}_{n}^{*-1} \mathbf{C}_{n}^{*}\right]^{-1}, \\
& \mathbf{D} \stackrel{\text { def }}{=}-\mathbf{A} \mathbf{C}_{n} \mathbf{R}_{n}^{*-1}
\end{aligned}
$$

Finally, substituting (24) into (17) and (18), we get, after some straightforward manipulations

$$
\operatorname{SINR}_{\mathrm{MMSE}}=\pi_{s}\left[\mathbf{s}^{H} \mathbf{A} \mathbf{s}\left(1+\left|\gamma_{s}\right|^{2}\right)+2 \operatorname{Re}\left(\gamma_{s}^{*} \mathbf{s}^{H} \mathbf{D} \mathbf{s}^{*}\right)-\frac{\left|\mathbf{s}^{H} \mathbf{D} \mathbf{s}^{*}+\gamma_{s} \mathbf{s}^{H} \mathbf{A} \mathbf{s}\right|^{2}}{\left[\pi_{s}\left(1-\left|\gamma_{s}\right|^{2}\right)\right]^{-1}+\mathbf{s}^{H} \mathbf{A} \mathbf{s}}\right]
$$

and

$$
\operatorname{SINR}_{\mathrm{MVDR}_{1}}=\pi_{s}\left[\mathbf{s}^{H} \mathbf{A} \mathbf{s}-\frac{\left|\mathbf{s}^{H} \mathbf{D} \mathbf{s}^{*}\right|^{2}}{\mathbf{s}^{H} \mathbf{A} \mathbf{s}}\right]
$$

The variations of SINR $\mathrm{MMSE}$ as a function of $\left|\gamma_{s}\right|$ are analyzed in the next section.

3) SINR analysis:

In the particular case of a SO circular SOI $\left(\gamma_{s}=0\right),(27)$ reduces to

$$
\operatorname{SINR}_{\mathrm{MMSE}}=\pi_{s}\left[\mathbf{s}^{H} \mathbf{A} \mathbf{s}-\frac{\left|\mathbf{s}^{H} \mathbf{D} \mathbf{s}^{*}\right|^{2}}{\pi_{s}^{-1}+\mathbf{s}^{H} \mathbf{A} \mathbf{s}}\right] ; \quad \gamma_{s}=0,
$$

which is always greater than $\mathrm{SINR}_{\mathrm{MVDR}_{1}}$, itself greater than $\mathrm{SINR}_{\mathrm{CAPON}}$ for SO noncircular total noise, and which tends to $\mathrm{SINR}_{\mathrm{MVDR}_{1}}$ as $\pi_{s} \mathbf{s}^{H} \mathbf{A} \mathbf{s} \gg 1$. Hence the interest of $\widetilde{\mathbf{w}}_{\mathrm{MMSE}}$ for a SO non circular total noise, even for a SO circular SOI.

In the particular case of a rectilinear SOI $\left(\left|\gamma_{s}\right|=1\right)$, (27) reduces to

$$
\operatorname{SINR}_{\mathrm{MMSE}}=2 \pi_{s}\left[\mathbf{s}^{H} \mathbf{A} \mathbf{s}-\left|\mathbf{s}^{H} \mathbf{D s}^{*}\right| \cos 2 \psi\right] ; \quad\left|\gamma_{s}\right|=1,
$$

where $\psi \stackrel{\text { def }}{=}\left(\phi_{d_{s}}-2 \phi_{s}+\pi\right) / 2$ and where $\phi_{d_{s}}$ is the phase of $d_{s} \stackrel{\text { def }}{=} \mathbf{s}^{H} \mathbf{D s}^{*}=\left|d_{s}\right| e^{i \phi_{d_{s}}}$. Expression (30) is nothing else than the SINR at the output of the WL beamformer analyzed in [11], which allows SAIC of one rectilinear interference.

Finally, for SO noncircular nonrectilinear SOI, i.e., for arbitrary values of $\left|\gamma_{s}\right|$ such that $0<\left|\gamma_{s}\right|<1$, two cases corresponding to $d_{s}=0$ and $d_{s} \neq 0$ must be considered.

When $d_{s}=0$, which occurs for a SO circular total noise $\left(\mathbf{C}_{n}=\mathbf{0}\right)$ or when $\mathbf{s}$ is in the kernel of $\mathbf{D}^{*}$,

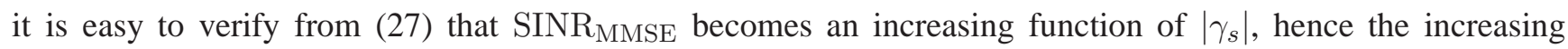
interest of $\widetilde{\mathbf{w}}_{\mathrm{MMSE}}$ as $\left|\gamma_{s}\right|$ increases. The minimum value of $\mathrm{SINR}_{\mathrm{MMSE}}$, obtained for $\gamma_{s}=0$, corresponds to $\pi_{s} \mathbf{s}^{H} \mathbf{R}_{n}^{-1} \mathbf{s}=$ SINR $_{\mathrm{CAPON}}$ whereas the maximum value of $\operatorname{SINR}_{\mathrm{MMSE}}$ is obtained for $\left|\gamma_{s}\right|=1$ and corresponds 
to $2 \pi_{s} \mathbf{s}^{H} \mathbf{R}_{n}^{-1} \mathbf{s}=2$ SINR $_{\mathrm{CAPON}}$, hence a maximal gain of $3 \mathrm{~dB}$ with respect to the Capon's beamformer obtained for rectilinear SOI.

When $d_{s} \neq 0$, which occurs for a SO noncircular total noise $\left(\mathbf{C}_{n} \neq \mathbf{0}\right)$ provided that $\mathbf{s}$ is not in the kernel of $\mathbf{D}^{*}$, it can be shown that, for $\cos 2 \psi \leq 0$ (i.e., $-\pi / 2+2 k \pi \leq \phi_{d_{s}}-2 \phi_{s} \leq \pi / 2+2 k \pi$, with $k$ integer), SINR $_{\text {MMSE }}$ becomes an increasing function of $\left|\gamma_{s}\right|$ lower and upper-bounded by (29) and (30) respectively, hence the increasing interest of $\widetilde{\mathbf{w}}_{\mathrm{MMSE}}$ as $\left|\gamma_{s}\right|$ increases. However, for $\cos 2 \psi>0$ (i.e., $\pi / 2+2 k \pi \leq \phi_{d_{s}}-2 \phi_{s} \leq$ $3 \pi / 2+2 k \pi$, with $k$ integer), there exists a value of $\left|\gamma_{s}\right|$, noted $\left|\gamma_{s, \min }(\psi)\right|$, such that $\operatorname{SINR}_{\mathrm{MMSE}}$ is a decreasing function of $\left|\gamma_{s}\right|$ for $0 \leq\left|\gamma_{s}\right| \leq\left|\gamma_{s, \min }(\psi)\right|$ and an increasing function of $\left|\gamma_{s}\right|$ for $\left|\gamma_{s, \min }(\psi)\right| \leq\left|\gamma_{s}\right| \leq 1$. This shows in this case the existence of a noncircularity coefficient modulus $\left|\gamma_{s, \min }(\psi)\right|>0$ which minimizes SINR $_{\text {MMSE}}$, which could seem a bit surprising and which shows, in this case, the increasing interest of $\widetilde{\mathbf{w}}_{\text {MMSE }}$ as $\left|\gamma_{s}\right|$ moves in the vicinity of either 0 or 1 .

To get more insights into the practical interest of the WL MMSE beamformer with respect to the Capon's and the WL MVDR 1 beamformers, we analyse, in the next section, for arbitrary SO noncircular SOI, performance of the three previous beamformers, in terms of maximal number of interference to be processed, output SINR and SER, in the presence of potentially noncircular rectilinear and nonrectilinear interferences plus background noise.

IV. Performance of the WL MMSE BEAMFormer IN THE PRESENCE OF NONCIRCUlar SOI AND/OR INTERFERENCES

\section{A. Total noise model}

We assume in this section that the total noise, $\mathbf{n}(t)$, is composed of $P$ statistically uncorrelated and potentially SO noncircular NB interferences plus background noise. Under these assumptions, the total noise vector $\mathbf{n}(t)$ can be written as follows

$$
\mathbf{n}(t)=\sum_{p=1}^{P} m_{p}(t) \mathbf{j}_{p}+\mathbf{n}_{b}(t),
$$

where $\mathbf{n}_{b}(t)$ is the background noise vector, assumed zero-mean, stationary, SO circular, Gaussian and spatially white; $m_{p}(t)$ and $\mathbf{j}_{p}$ correspond to the complex amplitude, assumed potentially SO noncircular and the steering or channel vector (whose first component is equal to one) of interference $p$, respectively. Under these assumptions, matrices $\mathbf{R}_{n}$ and $\mathbf{C}_{n}$, can be written as

$$
\begin{aligned}
\mathbf{R}_{n} & =\sum_{p=1}^{P} \pi_{p} \mathbf{j}_{p} \mathbf{j}_{p}^{H}+\eta_{2} \mathbf{I} \\
\mathbf{C}_{n} & =\sum_{p=1}^{P} \pi_{p} \gamma_{p} \mathbf{j}_{p} \mathbf{j}_{p}^{T}
\end{aligned}
$$


where $\eta_{2}$ is the mean power of the background noise per sensor; $\mathbf{I}$ is the $N \times N$ identity matrix; $\pi_{p} \stackrel{\text { def }}{=}<$ $\mathrm{E}\left[\left|m_{p}(t)\right|^{2}\right]>$ is the time averaged power of interference $p$ received by the first sensor; $\gamma_{p} \stackrel{\text { def }}{=}<\mathrm{E}\left[m_{p}^{2}(t)\right]>$ $/ \pi_{s}=\left|\gamma_{p}\right| e^{2 i \phi_{p}}$ such that $0 \leq\left|\gamma_{p}\right| \leq 1$, is the time averaged SO noncircularity coefficient of interference $p$.

\section{B. Maximal number of interferences to be processed}

We deduce from (9) that a nonrectilinear SOI generates one interference in $\widetilde{\mathbf{x}}(t)$, whereas a rectilinear SOI does not generate any interference in $\widetilde{\mathbf{x}}(t)$. Applying (9) to interference $p$, we deduce in a same way that a nonrectilinear interference $p$ generates two uncorrelated interferences in $\widetilde{\mathbf{x}}(t)$, whereas a rectilinear interference $p$ only generates one interference in $\widetilde{\mathbf{x}}(t)$. As a consequence, noting $P_{r}$ and $P_{n r}$ the number of rectilinear and nonrectilinear interferences in $\mathbf{x}(t)$, respectively, such that $P_{r}+P_{n r}=P$, the WL MMSE beamformer (with its interpretation as a WL MVDR beamformer subject to a single constraint) has then $2 N-1$ degrees of freedom to reject $2 P_{n r}+P_{r}+1-\delta\left(1-\left|\gamma_{s}\right|\right)$ interferences in $\widetilde{\mathbf{x}}(t)$, where $\delta($.$) is the Kronecker symbol$ such that $\delta(x)=1$ if $x=0$ and $\delta(x)=0$ if $x \neq 0$. Hence, the maximal number of interferences, $P_{\max }$, that may be rejected (or completely nulled in the absence of noise) by the WL MMSE beamformer is such that $2 N-1=2 P_{n r}+P_{r}+1-\delta\left(1-\left|\gamma_{s}\right|\right)$, which gives, with $P_{\max }=P_{r}+P_{n r}$ :

$$
P_{\max }=N-1+\left(P_{r}+\delta\left(1-\left|\gamma_{s}\right|\right)\right) / 2 \quad \text { with } 0 \leq P_{r} \leq P_{\max }
$$

and which means that

$$
N-1 \leq P_{\max } \leq 2(N-1)+\delta\left(1-\left|\gamma_{s}\right|\right) \leq 2 N-1
$$

In particular $P_{\max }$ is minimal and equal to $N-1$ whatever the noncircularity property of the received sources if at most one source (SOI or interference) is rectilinear. In this case, the exploitation of the potential SO noncircularity of the sources, through the use of the WL MMSE beamformer instead of the Linear MMSE or the Capon's beamformer, does not allow an increase of the number of interferences to be processed. This increase is possible and $P_{\max }>N-1$ only if at least two sources (SOI and/or interferences) are rectilinear. This increase is maximal if all the interferences are rectilinear. In this case, $P_{\max }=2(N-1)$ for a nonrectilinear SOI and $P_{\max }=2 N-1$ for a rectilinear SOI, which shows that the WL MMSE beamformer allows SAIC $\left(P_{\max }=1\right.$ for $\left.N=1\right)$ only if the SOI is rectilinear in the presence of a rectilinear interference, situation analyzed in detail in [11]. We deduce from this global analysis that the key property allowing an increase of the number of interferences to be processed by the WL MMSE beamformer with respect to the Capon's beamformer is the potential rectilinearity of the latter, and not simply their SO noncircularity, which shows off a first time the breakthrough between rectilinear and nonrectilinear interferences. 


\section{SINR for one and two interferences}

We analyse in this section, for rectilinear and nonrectilinear SOI, the practical interest of the WL MMSE beamformer in the presence of one and two strong noncircular nonrectilinear interferences through an analytical study of the output SINR (9).

1) Rectilinear SOI and one strong interference:

a) Strong rectilinear interference:

For a rectilinear SOI and one strong $\left(\epsilon_{1} \stackrel{\text { def }}{=}\left(\mathbf{j}_{1}^{H} \mathbf{j}_{1}\right) \pi_{1} / \eta_{2} \gg 1\right)$ rectilinear interference, (27) becomes :

$$
\operatorname{SINR}_{\mathrm{MMSE}} \approx 2 \epsilon_{s}\left(1-\left|\alpha_{1, s}\right|^{2} \cos ^{2} \psi\right) ; \quad \epsilon_{1} \gg 1,\left|\gamma_{s}\right|=\left|\gamma_{1}\right|=1
$$

whereas $\mathrm{SINR}_{\mathrm{CAPON}}$ and $\mathrm{SINR}_{\mathrm{MVDR}_{1}}$ are given by

$$
\begin{array}{lll}
\operatorname{SINR}_{\mathrm{CAPON}} & \approx \epsilon_{s}\left(1-\left|\alpha_{1, s}\right|^{2}\right) ; & \epsilon_{1} \gg 1,\left|\alpha_{1, s}\right| \neq 1, \forall \gamma_{s}, \forall \gamma_{1}, \\
\mathrm{SINR}_{\mathrm{MVDR}_{1}} \approx \epsilon_{s}\left(1-\frac{\left|\alpha_{1, s}\right|^{2}}{2-\left|\alpha_{1, s}\right|^{2}}\right) ; & \epsilon_{1} \gg 1,\left|\gamma_{1}\right|=1,\left|\alpha_{1, s}\right| \neq 1, \forall \gamma_{s} .
\end{array}
$$

In these expressions, $\epsilon_{s} \stackrel{\text { def }}{=}\left(\mathbf{s}^{H} \mathbf{s}\right) \pi_{s} / \eta_{2}, \alpha_{1, s} \stackrel{\text { def }}{=} \mathbf{j}_{1}^{H} \mathbf{s} /\left(\mathbf{s}^{H} \mathbf{s}\right)^{1 / 2}\left(\mathbf{j}_{1}^{H} \mathbf{j}_{1}\right)^{1 / 2}$ and $\psi$, appearing in (30), takes the value $\psi=\left[\phi_{1}-\phi_{s}+\operatorname{Arg}\left(\mathbf{s}^{H} \mathbf{j}_{1}\right)\right]$. Expression (36), which has also been obtained in [11], shows that in this case, the WL MMSE beamformer discriminates the sources both spatially (for $N>1$ ) and by phase, allowing in particular SAIC contrary to WL MVDR 1 and Capon's beamformers which discriminate sources spatially only (for $N>1$ ).

b) Strong nonrectilinear interference:

For a rectilinear SOI and one strong nonrectilinear interference $\left(\left|\gamma_{1}\right| \neq 1\right)$, provided that $\left|\alpha_{1, s}\right| \neq 1$, $\mathrm{SINR}_{\mathrm{MVDR}_{1}} \approx \mathrm{SINR}_{\mathrm{CAPON}}$ given by (37), whereas $\mathrm{SINR}_{\mathrm{MMSE}} \approx 2 \mathrm{SINR}_{\mathrm{CAPON}}$. In this case, $\mathrm{SINR}_{\mathrm{MMSE}}$ is twice the SINR at the output of Capon's and WL MVDR 1 beamformer due to the exploitation of the SO rectilinearity of the SOI, but is not greater than the SINR at the output of the WL MMSE beamformer for a SO circular interference. This shows the relatively weak practical interest to take into account the potential SO noncircularity property of a strong interference which is not rectilinear or not far from being rectilinear.

2) Nonrectilinear SOI and one strong interference:

a) Strong rectilinear interference:

For a nonrectilinear SOI and one strong rectilinear interference, $\mathrm{SINR}_{\mathrm{CAPON}}$ and $\mathrm{SINR}_{\mathrm{MVDR}_{1}}$ are still given by (37) and (38) respectively whereas we deduce from (24) to (26), (32) and (33) that SINR $_{\text {MMSE }}$ becomes

$$
\operatorname{SINR}_{\mathrm{MMSE}} \approx \epsilon_{s} \frac{\left(1+\left|\gamma_{s}\right|^{2}\right)\left(2-\left|\alpha_{1, s}\right|^{2}\right)-2\left|\gamma_{s}\right|\left|\alpha_{1, s}\right|^{2} \cos 2 \psi+2 \epsilon_{s}\left(1-\left|\gamma_{s}\right|^{2}\right)\left(1-\left|\alpha_{1, s}\right|^{2}\right)}{2+\epsilon_{s}\left(1-\left|\gamma_{s}\right|^{2}\right)\left(2-\left|\alpha_{1, s}\right|^{2}\right)}
$$

For a weak $\left(\epsilon_{s} \ll 1\right)$ nonrectilinear SOI, this expression reduces to:

$$
\operatorname{SINR}_{\mathrm{MMSE}} \approx \epsilon_{s}\left[\left(1+\left|\gamma_{s}\right|^{2}\right)\left(1-\left|\alpha_{1, s}\right|^{2} / 2\right)-\left|\gamma_{s}\right|\left|\alpha_{1, s}\right|^{2} \cos 2 \psi\right] ; \quad \epsilon_{1} \gg 1,\left|\gamma_{1}\right|=1, \epsilon_{s} \ll 1
$$


which shows, as already pointed out in Subsection III-D3 for $d_{s} \neq 0$, that when $\cos 2 \psi \leq 0$, SINR MMSE $_{2}$ is an increasing function of $\left|\gamma_{s}\right|$, lower-bounded by $\epsilon_{s}\left(1-\left|\alpha_{1, s}\right|^{2} / 2\right)$ obtained for $\gamma_{s}=0$, which is much more greater than $\operatorname{SINR}_{\mathrm{CAPON}}$ and $\mathrm{SINR}_{\mathrm{MVDR}_{1}}$ for high values of $\left|\alpha_{1, s}\right|^{2}$. This shows in this case the great interest to take into account the potential noncircularity of the SOI in addition to the rectilinear character of the interference. Moreover, when $\cos 2 \psi>0$, as also already pointed out in Subsection III-D3 for $d_{s} \neq 0$, their exists a value $\left|\gamma_{s, \min }(\psi)\right|$ of $\left|\gamma_{s}\right|$, given here by $\left|\gamma_{s, \min }(\psi)\right|=\left|\alpha_{1, s}\right|^{2} \cos 2 \psi /\left(2-\left|\alpha_{1, s}\right|^{2}\right)$, such that $\operatorname{SINR}_{\text {MMSE }}$ is a decreasing function of $\left|\gamma_{s}\right|$ for $0 \leq\left|\gamma_{s}\right| \leq\left|\gamma_{s, \min }(\psi)\right|$ and an increasing function of $\left|\gamma_{s}\right|$ for $\left|\gamma_{s, \min }(\psi)\right| \leq\left|\gamma_{s}\right| \leq 1$. Using $\left|\gamma_{s, \min }(\psi)\right|$ into (40), we deduce that in this case, SINR MMSE $_{\text {is lower-bounded }}$ by:

$$
\operatorname{SINR}_{\mathrm{MMSE}}\left[\left|\gamma_{s, \min }(\psi)\right|\right] \approx \epsilon_{s}\left(1-\left|\alpha_{1, s}\right|^{2} / 2\right)\left[1-\left|\alpha_{1, s}\right|^{4} \cos ^{2} 2 \psi /\left(2-\left|\alpha_{1, s}\right|^{2}\right)\right]
$$

which still corresponds to $\epsilon_{s}\left(1-\left|\alpha_{1, s}\right|^{2} / 2\right)$ for weak values of $\cos 2 \psi$ and which shows again, at least for high values of $\left|\alpha_{1, s}\right|^{2}$, the interest to take into account the potential noncircularity of the SOI in addition to the rectilinearity character of the interference.

For a strong $\left(\epsilon_{s} \gg 1\right)$ nonrectilinear SOI, expression (39) reduces to (38) whatever $\left|\gamma_{s}\right| \neq 1$ and $\left|\alpha_{1, s}\right| \neq 1$, which shows an increasing SINR gain toward $3 \mathrm{~dB}$ with respect to Capon's beamformer as $\left|\alpha_{1, s}\right|$ increases toward unity and which shows again the practical interest to take into account the rectilinear character of the strong interference.

\section{b) Strong nonrectilinear interference:}

Finally, for a nonrectilinear SOI and one strong nonrectilinear interference, $\mathrm{SINR}_{\mathrm{MVDR}_{1}} \approx \mathrm{SINR}_{\mathrm{CAPON}}$ given by (37), whereas we deduce from (24) to (26), (32) and (33) that $\operatorname{SINR}_{\mathrm{MMSE}}$ becomes:

$$
\operatorname{SINR}_{\mathrm{MMSE}} \approx \operatorname{SINR}_{\mathrm{CAPON}}\left(1+\frac{\left|\gamma_{s}\right|^{2}}{1+\epsilon_{s}\left(1-\left|\gamma_{s}\right|^{2}\right)\left(1-\left|\alpha_{1, s}\right|^{2}\right)}\right) ; \quad\left|\gamma_{s}\right| \neq 1,\left|\gamma_{1}\right| \neq 1, \epsilon_{1} \gg 1,\left|\alpha_{1, s}\right| \neq 1,
$$

which is an increasing function of $\left|\gamma_{s}\right|$ varying from SINR $_{\text {CAPON }}$ obtained for a SO circular SOI to 2SINR $_{\text {CAPON }}$ obtained for a rectilinear SOI. Nevertheless, for a strong nonrectilinear SOI such that $\epsilon_{s}\left(1-\left|\gamma_{s}\right|^{2}\right)\left(1-\left|\alpha_{1, s}\right|^{2}\right) \gg 1, \operatorname{SINR}_{\mathrm{MMSE}} \approx \mathrm{SINR}_{\mathrm{CAPON}}$, which shows the relatively weak practical interest to take into account the potential SO noncircularity property of both strong SOI and interference which are not rectilinear.

\section{3) Illustrations for one and two interferences:}

To illustrate the previous results related to the weak (resp., great) interest to take into account the potential noncircularity of a strong nonrectilinear (resp. rectilinear) interference, we consider that a uniform linear array (ULA) of $N$ omnidirectional sensors, equispaced half a wavelength apart, receives a background noise, a SOI and an interference whose directions of arrival (DOA) with respect to broadside are equal to $\theta_{s}$ and $\theta_{1}$ respectively. 
Under these assumptions, Figure 2 shows, for $N=2$, the variations of $\mathrm{SINR}_{\mathrm{CAPON}}, \mathrm{SINR}_{\mathrm{MVDR}_{1}}$ and $\operatorname{SINR}_{\mathrm{MMSE}}$ as a function of $\left|\gamma_{1}\right|$ for several values of $\left|\gamma_{s}\right|$ equal to 0, 0.5, 0.95 and 1 respectively. For this figure, $\pi_{s} / \eta_{2}=20 \mathrm{~dB}$ (strong SOI), $\pi_{1} / \eta_{2}=20 \mathrm{~dB}, \phi_{s}=0^{\circ}, \phi_{1}=60^{\circ}, \theta_{s}=0^{\circ}$ and $\theta_{1}=30^{\circ}$. Note increasing performance of the WL beamformers as $\left|\gamma_{1}\right|$ increases and the limited interest to take into account the noncircularity property of a nonrectilinear interference whatever the noncircularity property of the SOI. Note also the limited interest to take into account the SO noncircularity property of a nonrectilinear SOI, whatever the SO noncircularity property of the interference. Note also, even for $\left|\alpha_{1, s}\right| \neq 1$, very good performance and strong gains in performance of the WL MMSE beamformer with respect to Capon's beamformer when the SOI and interference are rectilinear with a sufficient phase discrimination between the sources, and a strong decrease of this gain as soon as one of the sources is no longer rectilinear.

Figure 3 shows the same variations as Figure 2 but for $\pi_{s} / \eta_{2}=-20 \mathrm{~dB}$ (weak SOI), $\pi_{1} / \eta_{2}=20 \mathrm{~dB}$, $\phi_{s}=0^{\circ}, \phi_{1}=45^{\circ}, \theta_{s}=0^{\circ}$ and $\theta_{1}=10^{\circ}$. As $\left|\alpha_{1, s}\right|^{2}$ is high, note strong performance gains of the WL MMSE beamformer with respect to $\operatorname{SINR}_{\mathrm{CAPON}}$ and $\mathrm{SINR}_{\mathrm{MVDR}_{1}}$ for high values of $\left|\gamma_{1}\right|$ whatever the value of $\left|\gamma_{s}\right|$. Note also the gain of $3 \mathrm{~dB}$ for a rectilinear SOI and nonrectilinear interferences and much weaker gain values for both nonrectilinear SOI and interference.

To complete these results, we consider the same scenario as for Figure 2, but for a SOI whose SNR is equal to $\pi_{s} / \eta_{2}=10 \mathrm{~dB}$, at which we add a second interference, assumed to be rectilinear and such that $\theta_{2}=60^{\circ}$, $\pi_{2} / \eta_{2}=20 \mathrm{~dB}$ and $\phi_{2}=80^{\circ}$. Under these assumptions, Figure 4 shows, for $N=2$, the same variations as for Figure 2. Note the poor performance of Capon's beamformer due to the overconstrained array. Note both very good performance and strong gain in performance of the two WL beamformers with respect to Capon's beamformer as soon as the two interferences are rectilinear. Note a strong decrease in performance of the two WL beamformers as soon as $\left|\gamma_{1}\right|$ decreases below 0.9, i.e. as soon as one of the two interferences strongly loses its rectilinear property. Nevertheless, despite the fact that the array is overconstrained, still note the not so bad performance of the WL MMSE beamformer with respect to the Capon's beamformer whatever $\left|\gamma_{1}\right|$ and $\left|\gamma_{s}\right|$ due to the presence of the rectilinear interference 2 .

\section{SER for interferences and background noise}

We show in this section that the main message of the previous section, deduced from an output SINR analysis and related to the weak interest to use WL MMSE beamformer for strong noncircular nonrectilinear interferences, remains valid from an output SER analysis. To this aim, we do some assumptions in Subsection IV-D1, we present the receivers used for demodulation in Subsection IV-D2, we compute output SER for BPSK SOI in Subsection IV-D3 and discuss and illustrate output SER for both BPSK and QPSK SOI in Subsection IV-D4.

\section{1) Hypotheses:}


a) Observation model:

To evaluate the SER performance at the output of the WL MMSE beamformer, we assume that the SOI is linearly digitally modulated and that a conventional Maximum Likelihood (ML) demodulator is inserted at the output of the WL MMSE beamformer before decision of the SOI symbols. For comparison perspectives, we also consider Capon's and WL MVDR 1 beamformers in addition to WL MMSE beamformer. To simplify the analysis, we assume that the SOI and the $P$ interferences are linearly modulated, have common $1 / 2$ Nyquist pulse shape filters, carriers and symbol rate and furthermore are perfectly synchronized. In the absence of frequency offsets, assuming an ideal symbol rate sampling, the sampled observation vectors at the output of a matched filter to the pulse shape filter can be written as:

$$
\mathbf{x}(k T)=\mu_{s} e^{i \zeta_{s}} a_{k} \mathbf{s}+\sum_{p=1}^{P} \mu_{p} e^{i \zeta_{p}} b_{p, k} \mathbf{j}_{p}+\mathbf{n}_{b}(k T),
$$

where $T$ is the symbol period, $\mathbf{x}(k T)$ is now the sampled observation vector at the output of the matched filter; $\mathbf{n}_{b}(k T)$ is the sampled background noise vector whose power is equal to $\eta_{2} ; a_{k}$ and $b_{p, k}$ are the symbols $k$ of SOI and interference $p(1 \leq p \leq P)$ respectively. Both $a_{k}$ 's and $b_{p, k}$ 's are assumed to be i.i.d. sequences with potentially different distributions, and $a_{k}, b_{i, q}$ and $b_{j, l}$ are statistically independent for $i \neq j ; \mu_{s}$ and $\mu_{p}$ are scalars which control the received power of the SOI and interference $p$, respectively; $\zeta_{s}$ and $\zeta_{p}$ are channel phase terms which control the received phase of the SOI and interference $p$ respectively. Defining $\pi_{a} \stackrel{\text { def }}{=} \mathrm{E}\left[\left|a_{k}\right|^{2}\right]$ and $\pi_{b, p} \stackrel{\text { def }}{=} \mathrm{E}\left[\left|b_{p, k}\right|^{2}\right]$ the power of SOI and interference $p$ symbol, respectively, we can write $\pi_{s}=\mu_{s}^{2} \pi_{a}$ and $\pi_{p}=\mu_{p}^{2} \pi_{b, p}$. Defining $\gamma_{a} \stackrel{\text { def }}{=} \mathrm{E}\left[a_{k}^{2}\right] / \pi_{a} \stackrel{\text { def }}{=}\left|\gamma_{a}\right| e^{2 i \phi_{a}}$ and $\gamma_{b, p} \stackrel{\text { def }}{=} \mathrm{E}\left[b_{p, k}^{2}\right] / \pi_{b, p} \stackrel{\text { def }}{=}\left|\gamma_{b, p}\right| e^{2 i \phi_{b, p}}$, we can write $\gamma_{s}=\gamma_{a} e^{2 i \zeta_{s}}=\left|\gamma_{a}\right| e^{2 i \phi_{s}}$, and $\gamma_{p}=\gamma_{p, b} e^{2 i \zeta_{p}}=\left|\gamma_{p}\right| e^{2 i \phi_{p}}$, hence $\left|\gamma_{s}\right|=\left|\gamma_{a}\right|,\left|\gamma_{p}\right|=\left|\gamma_{b, p}\right|, \phi_{s}=\phi_{a}+\zeta_{s}$ and $\phi_{p}=\phi_{b, p}+\zeta_{p}$.

\section{b) $U-V Q A M$ interferences:}

To limit the developments, we assume that the SOI may correspond to either a BPSK $\left(\left|\gamma_{s}\right|=1\right)$ or a QPSK $\left(\gamma_{s}=0\right)$ SOI. Moreover, to take into account in our SER analysis interferences having arbitrary values of SO noncircularity coefficient, we assume that interference $p$ corresponds to a rectangular $U-V$ QAM modulation with $U V$ states where integer $V$ may be even $(V=2 v)$ or odd $(V=2 v+1)$ whereas integer $U$ is necessarily even $(U=2 u)$. More precisely if $b_{k}=b_{r, k}+i b_{i, k}$ is a $U-V$ QAM symbol, where $b_{r, k}$ and $b_{i, k}$ are the real and imaginary part of $b_{k}$ respectively, assumed to be statistically independent to each other, then $b_{r, k}$ may take the values $\pm 1, \pm 3, \ldots, \pm(2 u-1)$ whereas $b_{i, k}$ may take the values $\pm 1, \pm 3, \ldots, \pm(2 v-1)$ if $V=2 v$ and the values $0, \pm 2, \pm 4, \ldots, \pm 2 v$ if $V=2 v+1$. In particular, a $U-1$ QAM modulation is a rectilinear modulation corresponding to a $U$-ASK modulation, which reduces to a BPSK modulation for $U=2$. A 2-2 QAM modulation is a SO circular modulation corresponding to a QPSK modulation. It is straightforward to prove, after elementary computations which are not reported here, that the power, $\pi_{b}$, of a $U-V$ QAM symbol $b_{k}$ is 
such that

$$
\begin{array}{ll}
\pi_{b}=\left[4\left(u^{2}+v^{2}\right)-2\right] / 3 & \text { for } U=2 u \text { and } V=2 v, \\
\pi_{b}=\left[4 u^{2}+4 v(v+1)-1\right] / 3 & \text { for } U=2 u \text { and } V=2 v+1
\end{array}
$$

whereas the SO noncircularity coefficient, $\gamma_{b}$, of a $U-V$ QAM symbol $b_{k}$ is such that

$$
\begin{array}{ll}
\gamma_{b}=\frac{2\left(u^{2}-v^{2}\right)}{2\left(u^{2}+v^{2}\right)-1} & \text { for } U=2 u \text { and } V=2 v \\
\gamma_{b}=\frac{4 u^{2}-4 v(v+1)-1}{4 u^{2}+4 v(v+1)-1} & \text { for } U=2 u \text { and } V=2 v+1 .
\end{array}
$$

Expressions (46) and (47), which are new, show that it is possible to generate values of $\gamma_{b}$ comprised between 0 and 1 by appropriately choosing $U$ and $V$. This will be useful to generate noncircular nonrectilinear interferences for the computer simulations considered in Subsection IV-D4.

2) Receivers:

To present more in details the different receivers used in this section for demodulation purpose, we compute, from (43), the sampled extended observation vector defined by

$$
\widetilde{\mathbf{x}}(k T)=\mu_{s} e^{i \zeta_{s}} a_{k} \widetilde{\mathbf{s}}_{1}+\mu_{s} e^{-i \zeta_{s}} a_{k}^{*} \widetilde{\mathbf{s}}_{2}+\sum_{p=1}^{P} \mu_{p} e^{i \zeta_{p}} b_{p, k} \widetilde{\mathbf{j}}_{p, 1}+\sum_{p=1}^{P} \mu_{p} e^{-i \zeta_{p}} b_{p, k}^{*} \widetilde{\mathbf{j}}_{p, 2}+\widetilde{\mathbf{n}}_{b}(k T),
$$

where $\widetilde{\mathbf{n}}_{b}(k T) \stackrel{\text { def }}{=}\left[\mathbf{n}_{b}^{T}(k T), \mathbf{n}_{b}^{H}(k T)\right]^{T}, \widetilde{\mathbf{j}}_{p, 1} \stackrel{\text { def }}{=}\left[\mathbf{j}_{p}^{T}, \mathbf{0}_{N}^{T}\right]^{T}$ and $\widetilde{\mathbf{j}}_{p, 2} \stackrel{\text { def }}{=}\left[\mathbf{0}_{N}^{T}, \mathbf{j}_{p}^{H}\right]^{T}$; The sampled output, $y(k T) \stackrel{\text { def }}{=}$ $\widetilde{\mathbf{w}}^{H} \widetilde{\mathbf{x}}(k T)$, of the WL beamformer $\widetilde{\mathbf{w}}$ is then given by

$$
y(k T)=\alpha_{1} a_{k}+\alpha_{2} a_{k}^{*}+\sum_{p=1}^{P} \beta_{p, 1} b_{p, k}+\sum_{p=1}^{P} \beta_{p, 2} b_{p, k}^{*}+n_{k},
$$

where $\alpha_{1} \stackrel{\text { def }}{=} \mu_{s} e^{i \zeta_{s}} \widetilde{\mathbf{w}}^{H} \widetilde{\mathbf{s}}_{1}, \alpha_{2} \stackrel{\text { def }}{=} \mu_{s} e^{-i \zeta_{s}} \widetilde{\mathbf{w}}^{H} \widetilde{\mathbf{s}}_{2}, \beta_{p, 1} \stackrel{\text { def }}{=} \mu_{p} e^{i \zeta_{p}} \widetilde{\mathbf{w}}^{H} \widetilde{\mathbf{j}}_{p, 1}, \beta_{p, 2} \stackrel{\text { def }}{=} \mu_{p} e^{-i \zeta_{p}} \widetilde{\mathbf{w}}^{H} \widetilde{\mathbf{j}}_{p, 2}$ and $n_{k} \stackrel{\text { def }}{=}$ $\widetilde{\mathbf{w}}^{H} \widetilde{\mathbf{n}}_{b}(k T)$. The conventional MLSE receiver for demodulation of $a_{k}$, built from (49), generates $\widehat{a}_{k}$ given by

$$
\widehat{a}_{k}=\operatorname{Arg}_{a_{k}} \operatorname{Min}\left[\left|y(k T)-\alpha_{1} a_{k}-\alpha_{2} a_{k}^{*}\right|^{2}\right],
$$

which gives, for BPSK SOI,

$$
\widehat{a}_{k}=\operatorname{Sign}\left(\operatorname{Re}\left[\alpha^{*} y(k T)\right]\right)
$$

where $\alpha=\alpha_{1}+\alpha_{2}$.

3) SER computation for a BPSK SOI:

The SER computation for a QPSK SOI is a bit tedious and we limit the analytical SER computation to BPSK SOI. Results for QPSK SOI will be presented in the next section from Monte Carlo Simulations. For a BPSK 
SOI, the input, $z(k T) \stackrel{\text { def }}{=} \operatorname{Re}\left[\alpha^{*} y(k T)\right]$, of the sign detector (51) is given by

$$
z(k T)=|\alpha|^{2} a_{k}+\sum_{p=1}^{P} \operatorname{Re}\left[\beta_{p} b_{p, k}\right]+n_{z, k},
$$

where $\beta_{p} \stackrel{\text { def }}{=} \alpha^{*} \beta_{p, 1}+\alpha \beta_{p, 2}^{*}$ and where $n_{z, k} \stackrel{\text { def }}{=} \operatorname{Re}\left[\alpha^{*} n_{k}\right]$ is zero-mean and Gaussian distributed with variance

$$
\sigma_{n, z}^{2}=\frac{\eta_{2}}{2}\left(|\alpha|^{2}|| \widetilde{\mathbf{w}} \|^{2}+\operatorname{Re}\left[\alpha^{* 2}\left(\mathbf{w}_{1}^{H} \mathbf{w}_{2}^{*}+\mathbf{w}_{2}^{H} \mathbf{w}_{1}^{*}\right)\right]\right),
$$

where $\widetilde{\mathbf{w}} \stackrel{\text { def }}{=}\left[\mathbf{w}_{1}^{T}, \mathbf{w}_{2}^{T}\right]^{T}$. Using the symmetry property of the considered $U-V$ QAM constellation, it can be shown, after some straightforward manipulations, that the SER at the output of the sign detector is given by

$$
\mathrm{SER}=\left(\prod_{p=1}^{P} \frac{1}{U_{p} V_{p}}\right) \sum_{\left(b_{1, k}, \ldots, b_{P, k}\right)} Q\left(\frac{|\alpha|^{2}-\sum_{p=1}^{P} \operatorname{Re}\left(\beta_{p} b_{p, k}\right)}{\sigma_{n, z}}\right)
$$

where $U_{p}$ and $V_{p}$ are integer such that interference $p$ is $U_{p}-V_{p}$ QAM modulated, $\left(b_{1, k}, \ldots, b_{P, k}\right)$ denotes all the $\prod_{p}^{P} \frac{1}{U_{p} V_{p}}$ interference $P$-uples symbols and where $Q(u)$ is the function defined by

$$
Q(u) \stackrel{\text { def }}{=} \int_{u}^{\infty} \frac{1}{\sqrt{2 \pi}} e^{-\frac{v^{2}}{2}} d v
$$

Note that for a single BPSK interference, (54) corresponds to expression (54) of [11].

4) SER Illustrations:

In contrast to Gaussian interference and linear receivers, the behavior of SINR and SER, computed in Subsection IV-C and IV-D3 respectively, are not directly related. The aim of this subsection is then to verify whether the results obtained in Subsection IV-C for output SINR are still valid for output SER.

For this purpose Figure 5 and 6 show, for a BPSK and a QPSK SOI respectively, the variations of the SER at the output of Capon, WL MVDR 1 and WL MMSE beamformers as a function of $\pi_{s} / \eta_{2}$, for different values of $\left(U_{1}, V_{1}\right)$, when the total noise is composed of a circular Gaussian background noise and one $U_{1}-V_{1}$ QAM interference such that $\pi_{1} / \eta_{2}=20 \mathrm{~dB}, \theta_{s}=0^{\circ}, \theta_{1}=30^{\circ}, \phi_{s}=0^{\circ}, \phi_{1}=60^{\circ}$ and when $N=2$ sensors equispaced half a wavelength apart. For BPSK SOI, the SER is computed from the closed-form expression (54) whereas for QPSK SOI the SER is computed from Monte Carlo simulations from 100000 realizations $^{2}$. The chosen values of $\left(U_{1}, V_{1}\right)$ correspond to $(2,2)\left(\gamma_{1}=0\right),(4,2)\left(\gamma_{1}=0.666\right),(6,2)\left(\gamma_{1}=0.804\right),(10,2)$ $\left(\gamma_{1}=0.941\right)$ and $(2,1)\left(\gamma_{1}=1\right)$. Figure 5 shows, for a rectilinear SOI, substantial performance gains of both the WL MMSE beamformer and the WL MVDR 1 beamformer with respect to Capon's beamformer as $\left|\gamma_{1}\right|$ approaches unity, displaying the practical interest to take into account the potential SO noncircularity property of both the SOI and a strong interference which are rectilinear or almost rectilinear. Note also in this case

\footnotetext{
${ }^{2}$ Note that for QPSK SOI, the MLSE receiver (50) gives four decision areas in the complex plane where the Gaussian noisy component is noncircular. Consequently the derived closed-form expression of the SER is composed of $2 D$ integrals with no engineering insights that can be numerically calculated by approximations, only
} 
similar performances of the two WL beamformers due to the use of the ML receiver from the output of these beamformers which takes a decision from the real part of the output in both cases. Moreover, Figure 5 shows almost similar performances of the three beamformers as $\left|\gamma_{1}\right|$ moves away from unity, displaying the relatively weak practical interest to take into account the potential SO noncircularity property of a strong noncircular interference which is not rectilinear or almost rectilinear. Same conclusions are obtained from Figure 6 for a SO circular SOI. These results finally show that the main conclusions related to the weak interest to use WL MMSE beamformers for strong noncircular nonrectilinear interferences done from the output SINR analysis are still valid from an output SER point of view.

\section{RECTILINEARITY AND QUASI-RECTILINEARITY BLIND DETECTION}

\section{A. Context}

It has been shown in the previous sections that there is no real interest to use WL MMSE beamforming when some kind of rectilinearity is not hidden in the observations. As a consequence of this result, the priority to evaluate the interest of WL MMSE beamforming is to try to detect the potential presence of rectilinear sources in the observations before beamforming processing. Following this work, the detection of rectilinearity, instead of SO noncircularity, thus becomes a new problem of practical interest which has not yet been investigated to the best of our knowledge and which remains completely open. Despite the fact that this problem is far from being trivial, we propose in this section a preliminary method to detect the presence of rectilinearity (or quasi-rectilinearity) in circularly Gaussian noisy observations. We first recall the observation model and the statistics used by the proposed method. Then we present a method able to detect a rectilinear source corrupted by potential other sources and background noise and we evaluate some of its performance. Finally we briefly discuss the problem of quasi-rectilinearity detection.

\section{B. Model and Statistics}

\section{1) Model:}

We assume in this section that the complex observation vector, $\mathbf{x}(t)$, is composed of $M$ statistically independent and potentially SO noncircular NB sources plus background noise. It corresponds to a noisy instantaneous mixtures of $M$ statistically independent, NB and potentially SO noncircular sources and can be written as:

$$
\mathbf{x}(t)=\sum_{m=1}^{M} s_{m}(t) \mathbf{a}_{m}+\mathbf{n}_{b}(t) \stackrel{\text { def }}{=} \mathbf{A} \mathbf{s}(t)+\mathbf{n}_{b}(t)
$$

where $\mathbf{n}_{b}(t)$ is the background noise vector, assumed to be zero-mean, stationary, SO circular, Gaussian and spatially white; $s_{m}(t)$ and $\mathbf{a}_{m}$ are the complex envelope (to within a potential frequency offset) and the steering (or channel) vector of the source $m$ respectively; A is the so-called $N \times M$ mixing matrix whose columns are the $\mathbf{a}_{m}$ vectors and $\mathbf{s}(t)$ is the so-called $M \times 1$ source vector whose components are the $s_{m}(t)(1 \leq m \leq$ 
$M)$. We denote by $M_{r}$ and $M_{n r}$ the number of rectilinear and nonrectilinear sources respectively such that $M_{r}+M_{n r}=M$. As we only propose a preliminary method to detect rectilinearity in the observation vector (56), we limit our analysis to overdetermined mixtures of sources for which $M \leq N$. The case of underdetermined mixtures of sources, for which $M>N$, requires further developments which are not presented in this paper.

\section{2) Statistics:}

The SO statistics of the observations which are exploited in the following correspond to the temporal mean of the first and second correlation matrices of $\mathbf{x}(t)$, defined by

$$
\begin{aligned}
& \mathbf{R}_{x} \stackrel{\text { def }}{=}<\mathrm{E}\left[\mathbf{x}(t) \mathbf{x}^{H}(t)\right]>=\mathbf{A R}_{s} \mathbf{A}^{H}+\eta_{2} \mathbf{I} \stackrel{\text { def }}{=} \mathbf{R}_{x_{s}}+\eta_{2} \mathbf{I} \\
& \mathbf{C}_{x} \stackrel{\text { def }}{=}<\mathrm{E}\left[\mathbf{x}(t) \mathbf{x}^{T}(t)\right]>=\mathbf{A} \mathbf{C}_{s} \mathbf{A}^{T},
\end{aligned}
$$

where $\eta_{2}$ is the power of the background noise per antenna, $\mathbf{R}_{s} \stackrel{\text { def }}{=}<\mathrm{E}\left[\mathbf{s}(t) \mathbf{s}^{H}(t)\right]>$ and $\mathbf{C}_{s} \stackrel{\text { def }}{=}<$ $\mathrm{E}\left[\mathbf{s}(t) \mathbf{s}^{T}(t)\right]>$, diagonal under the previous hypotheses, are the temporal mean of the first and second correlation matrix of $\mathbf{s}(t)$ respectively and $\mathbf{R}_{x_{s}} \stackrel{\text { def }}{=} \mathbf{A} \mathbf{R}_{s} \mathbf{A}^{H}$ is the temporal mean of the first correlation matrix of the mixed sources. Note that the elements $[i, i]$ of matrices $\mathbf{R}_{s}$ and $\mathbf{C}_{s}$, denoted by $\mathbf{R}_{s}[i, i]$ and $\mathbf{C}_{s}[i, i]$ respectively, are such that $\mathbf{R}_{s}[i, i]$ is the input power of the source $i$ per omnidirectional antenna, denoted by $\pi_{i}$, and $\mathbf{C}_{s}[i, i]=\gamma_{i} \mathbf{R}_{s}[i, i]$, where $\gamma_{i}$ is the SO noncircularity coefficient of source $i$.

In a same way, the fourth order (FO) statistics of the observations which are exploited in the following correspond to the temporal mean, $\mathbf{Q}_{x}$, of the first quadricovariance matrix of $\mathbf{x}(t)$ whose elements are defined by $\mathbf{Q}_{x}[i, j, k, l] \stackrel{\text { def }}{=}<\operatorname{Cum}\left(x_{i}(t), x_{j}^{*}(t), x_{k}^{*}(t), x_{l}(t)\right)>$. Using (56) and assuming that $\mathbf{Q}_{x}[i, j, k, l]$ is the element $[N(i-1)+j, N(k-1)+l]$ of matrix $\mathbf{Q}_{x}$, we obtain the expression of the latter, given, under the previous assumptions, by

$$
\mathbf{Q}_{x}=\left(\mathbf{A} \otimes \mathbf{A}^{*}\right) \mathbf{Q}_{s}\left(\mathbf{A} \otimes \mathbf{A}^{*}\right)^{H}=\sum_{m=1}^{M} c_{m}\left(\mathbf{a}_{m} \otimes \mathbf{a}_{m}^{*}\right)\left(\mathbf{a}_{m} \otimes \mathbf{a}_{m}^{*}\right)^{H},
$$

where $\mathbf{Q}_{s}$ is the temporal mean of the first quadricovariance matrix of $\mathbf{s}(t), \quad c_{i} \stackrel{\text { def }}{=}<$ $\operatorname{Cum}\left(s_{i}(t), s_{i}^{*}(t), s_{i}^{*}(t), s_{i}(t)\right)>$ and $\otimes$ corresponds to the Kronecker product.

\section{A preliminary method to detect rectilinearity}

\section{1) Philosophy of the method:}

The philosophy proposed in this paper to blindly detect a rectilinear source in the observation (56) from a given number of observation snapshots $K$ consists, for each value of the source number estimate, $\widehat{M}$, comprised between 1 and $N$ (since $M$ is not known a priori):

- to blindly separate the $\widehat{M}$ mixed sources which are assumed to be present in $\mathbf{x}(t)$, from a wellknown blind source separation method of instantaneous mixture of sources developed these last twenty years [20], 
- to estimate the SO noncircularity coefficients, $\widehat{\gamma}_{o, i}$, of all the outputs $i(1 \leq i \leq \widehat{M})$ of the associated separator;

- to compare, for each separator output $i(1 \leq i \leq \widehat{M}),\left|\widehat{\gamma}_{o, i}\right|$ to a threshold $\beta(K, \widehat{M})$ whose value, which depends on $K$ and $\widehat{M}$, the chosen separator and the noisy mixture of sources, is a function of the false alarm rate we have chosen a priori;

- to declare detection of rectilinearity when one $\left|\widehat{\gamma}_{o, i}\right|$ is beyond the threshold $\beta(K, \widehat{M})$.

2) Steps of the method:

The mathematical steps of the method described previously are summarized hereafter :

- Initialisation of the number of source estimate : $\widehat{M}=1$

- Blind source separation of the $\widehat{M}$ mixed sources which are assumed to be present in $\mathbf{x}(t)$. We choose here the JADE method presented in [5] whose different steps are summarized hereafter:

- Empirical sample estimation, $\widehat{\mathbf{R}}_{x}(K)$ [15], of $\mathbf{R}_{x}$ from the $K$ observation snapshots $\mathbf{x}\left(k T_{e}\right),(1 \leq$ $k \leq K)$, where $T_{e}$ is the sample period;

- Prewhitening of the observation vectors $\mathbf{x}\left(k T_{e}\right),(1 \leq k \leq K)$, by the pseudo-inverse, $\widehat{\mathbf{F}}$, of a squareroot of $\widehat{\mathbf{R}}_{x_{s}}(K)$, an empirical estimate of $\mathbf{R}_{x_{s}}$ of rank $\widehat{M}$, computed from $\widehat{\mathbf{R}}_{x}(K)$ and $\widehat{M}$. This pre-whitening operation aims at orthonormalizing the source steering vectors so as to search for the latter through a unitary matrix $\mathbf{U}$, simpler to handle. We denote by $z\left(k T_{e}\right) \stackrel{\text { def }}{=} \widehat{\mathbf{F}} \mathbf{x}\left(k T_{e}\right),(1 \leq k \leq K)$, the $K \widehat{M} \times 1$ whitened sampled observation vectors;

- Empirical estimation, $\widehat{\mathbf{Q}}_{z}(K)$ [15], from the $K$ observation snapshots $z\left(k T_{e}\right),(1 \leq k \leq K)$, of $\mathbf{Q}_{z}$, the first quadricovariance matrix of $\mathbf{z}(t)$ );

- Blind identification, by $\widehat{\mathbf{U}}$, of the $\mathbf{U}$ matrix so as to jointly diagonalize the $\widehat{M}$ weighted eigenmatrices of $\widehat{\mathbf{Q}}_{z}(K)$, associated with the $\widehat{M}$ eigenvalues having the greatest modulus, where the weights correspond to the eigenvalues themselves;

- Blind identification, $\widehat{\mathbf{A}}$, of the mixing matrix $\mathbf{A}$ from $\widehat{\mathbf{U}}$ and $\widehat{\mathbf{F}}$;

- Building of the $N \times \widehat{M}$ blind source separator $\widehat{\mathbf{W}}=\widehat{\mathbf{R}}_{x}^{-1}(K) \widehat{\mathbf{A}}$ and generation of the $K \widehat{M} \times 1$ output vectors $y\left(k T_{e}\right)=\widehat{\mathbf{W}}^{H} \mathbf{x}\left(k T_{e}\right),(1 \leq k \leq K)$, containing, for each sample $k$, the $\widehat{M}$ separated sources respectively. The component $i$ of $\mathbf{y}\left(k T_{e}\right)$ is denoted by $y_{i}\left(k T_{e}\right)$.

- SO noncircularity coefficient estimation of the outputs of the separator: For each output $i,(1 \leq i \leq \widehat{M})$, of the separator $\widehat{\mathbf{W}}$, estimation, from the K output samples $y_{i}\left(k T_{e}\right)(1 \leq k \leq K)$, of the SO noncircularity coefficient of $y_{i}$ by $\widehat{\gamma}_{o, i}$ defined by

$$
\widehat{\gamma}_{o, i}=\sum_{k=1}^{K} y_{i}^{2}\left(k T_{e}\right) / \sum_{k=1}^{K}\left|y_{i}^{2}\left(k T_{e}\right)\right|
$$

- Potential detection of rectilinearity: 
- Choice of a threshold, $\beta(K, \widehat{M})$, ensuring a given false alarm rate

- If $\widehat{\mid} \gamma_{o, i} \mid>\beta(K, \widehat{M})$ for a particular value of $i$ :

$\circ$ Rectilinearity detection

$\circ$ End of the method

- If $\widehat{\mid} \gamma_{o, i} \mid \leq \beta(K, \widehat{M})$ for all $i(1 \leq i \leq \widehat{M})$ and if $\widehat{M}<N$ :

- Replace $\widehat{M}$ by $\widehat{M}+1$ and reiteration of the steps of the method

- If $\left|\gamma_{o, i}\right| \leq \beta(K, \widehat{M})$ for all $i(1 \leq i \leq \widehat{M})$ and if $\widehat{M}=N$ :

$\circ$ No rectilinearity detection

$\circ$ End of the method

\section{3) Performance of the method:}

To briefly illustrate the performance of the proposed method, we consider that a uniform linear array (ULA) of $N$ omnidirectional sensors, equispaced half a wavelength apart, receives a background noise and two statistically independent NB sources. The sources are either BPSK (rectilinear) or QPSK (SO circular) sources having the same symbol duration $T$ and the same raised cosine pulse shaped filter with a roll-off $\mu$. The two sources are synchronized to each other. The source $m(m=1,2)$ has a DOA with respect to broadside equal to $\theta_{m}$, a phase on the first sensor equal to $\phi_{m}$ and a Signal to Noise ratio per antenna equal to $\pi_{m} / \eta_{2}$. Three scenarios corresponding to (QPSK, QPSK), (BPSK, QPSK) and (BPSK, BPSK) sources respectively are considered in the following. For all of these scenarios, $N=2, T_{e}=T, \mu=0.3, \theta_{1}=30^{\circ}, \theta_{2}=80^{\circ}, \phi_{1}=40^{\circ}, \phi_{2}=80^{\circ}$, $\pi_{1} / \eta_{2}=\pi_{2} / \eta_{2}=10 \mathrm{~dB}$. Note that the SNR of the sources are chosen not too strong to verify the ability of the proposed method to detect rectilinearity in the observations even in relatively difficult situations.

Under these assumptions, Tables 1 to 3 show, for one realization of each of the three previous scenarios, for several values of $K$ and for all the possible values of $\widehat{M}$ (i.e., $\widehat{M}=1$ or 2 ), the maximum value of $\widehat{\mid} \gamma_{o, i} \mid$ over all the outputs of the separator $(1 \leq i \leq \widehat{M})$, denoted by $\left|\widehat{\gamma}_{o, \max }(K, \widehat{M})\right|$. For each couple $(K, \widehat{M})$, we note a relatively weak value of $\left|\widehat{\gamma}_{o, \max }(K, \widehat{M})\right|$ with respect to one for scenario 1 , for which no rectilinear source is present, and a value of $\left|\widehat{\gamma}_{o, \max }(K, \widehat{M})\right|$ greater than 0.87 for $\widehat{M}=2$ for scenario 2 and 3, i.e., in the presence of at least one rectilinear source. These results thus show, for each couple $(K, \widehat{M})$, the existence of a high contrast of values $\left|\widehat{\gamma}_{o, \max }(K, \widehat{M})\right|$ between scenario 1 and scenarios 2 and 3, even increasing with relatively high values of $K(K \geq 100)$ and $\widehat{M}=2$. This high contrast of values $\left|\widehat{\gamma}_{o, \text { max }}(K, \widehat{M})\right|$ between the absence and the presence of rectilinear sources jointly with a thinner analysis, out of the scope of this paper, would allow to choose, for each couple $(K, \widehat{M})$, a threshold $\beta(K, \widehat{M})$ ensuring a good probability of rectilinearity detection for scenarios 2 and 3, a poor probability of rectilinearity detection for scenario 1 jointly with a relatively low false alarm rate in all cases, hence the interest of the proposed method. 


\section{A preliminary method to detect quasi-rectilinearity}

The philosophy proposed in this paper to detect a rectilinearity in the observation (56) can be extended to detect quasi-rectilinear sources such as MSK, GMSK or OQAM sources to within some adaptations of the method. Indeed a quasi-rectilinear source is a source which can be written as a filtered version of a rectilinear source after a derotation operation by $i^{-t / T}$, where $T$ is the symbol duration for MSK and GMSK sources and half the symbol duration of the associated QAM modulation for OQAM source (see [11], [31]). In this context, the method proposed in Section V-C has to be adapted to take into account both the derotation and the filtering operation.

The derotation operation requires the a priori estimation of the symbol rate of each source, which correspond to the first non zero cyclic frequency of the first correlation function of the source. Thus, a first adaptation of the method is to implement a first step of cyclic frequency detection in the first correlation matrix of the observations. Such cyclic detectors have been proposed for example in [16] by Gardner. Once these cyclic frequencies of the observations have been estimated, the possible symbol durations of the sources are available. For each of these possibilities, corresponding derotated observations (for potential MSK, GMSK or OQAM modulations) may be built and may correspond to the inputs of the method proposed in Section V-C.

The filtering operation requires the use of blind source separators able to process convolutive mixtures of cyclostationary sources instead of instantaneous mixtures. For this reason, separators such as JADE become useless and have to be replaced by more sophisticated sources separators such as those developed in [18], [19].

With these modifications, the philosophy developed in Section V-C may still be used to detect quasirectilinearity in the observations but at the price of a higher complexity.

\section{SYNTHESIS AND CONCLUSION}

In this paper, enlightening properties and performance, in terms of output SINR and SER after demodulation, of the Time Invariant (TI) WL MMSE beamformer in the presence of SO noncircular SOI and/or interferences which are not necessarily rectilinear have been presented in a selfcontained, coherent, unified, guided and progressive way. One property of the WL MMSE beamformer concerns its collinearity with a WL MVDR beamformer (called WL $\mathrm{MVDR}_{2}$ ) which steers a beam in the virtual direction of an extended steering vector $\widetilde{\mathbf{s}}_{\gamma}$ which depends on both the true channel or steering vector, s, and the SO non circularity coefficient, $\gamma_{s}$, of the SOI. An equivalent GSC structure of this WL MVDR 2 bemformer has been described in the paper. This allows in particular to implement the WL MMSE beamformer from either the knowledge of a training sequence which is correlated with the SOI and not correlated with the total noise (as in radiocommunications) or, to within a constant, the knowledge or the estimation of both $\mathbf{s}$ and $\gamma_{s}$ (as for spectrum monitoring applications). Besides, under the constraint $\widetilde{\mathbf{w}}^{H} \widetilde{\mathbf{S}}_{\gamma}=1$, it has been shown in the paper that SINR maximization and MSE minimization are equivalent criteria which both generates the WL MVDR 2 beamformer. Despite 
the fact that the WL MMSE beamformer always increases the output performance with respect to the linear MMSE beamformer (or the Capon's beamformer) for SO noncircular SOI and/or interferences, whatever the number $N$ of antennas, it increases the number of sources to be processed beyond $N-1$ only when at least two of the received sources (SOI and/or interferences) are rectilinear. In particular, it allows SAIC in the presence of a rectilinear SOI and one rectilinear interference. These results confirm in particular the wellknown practical interest to take into account the potential SO noncircularity property of a rectilinear or an almost rectilinear interference. However a performance analysis in the presence of either arbitrary SO noncircular or rectangular QAM modulated interferences, in terms of both output SINR and SER, shows, at least for a strong SOI, the relatively weak practical interest of the WL MMSE beamformer for strong noncircular nonrectilinear interferences. This breakthrough between rectilinear and noncircular nonrectilinear strong interferences, which does not seem to be well-known by the scientific community, thus generates a new open problem for the choice between linear and WL MMSE receiver corresponding to the detection of rectilinearity (or quasi-rectilinearity), instead of noncircularity, in a given noisy observation. Although this question is mainly out of the scope of this paper, we have proposed preliminary tools based on blind source separation methods to solve this problem. An other consequence of the previous breakthrough for radio communications networks using rectilinear or quasirectilinear modulations associated with optimal TI WL beamformers, is that it is then crucial to estimate and to compensate, with a precision depending on the training sequence and burst duration, the different frequency offsets of the source before their processing. This precision has been evaluated precisely and recently in [14] for BPSK links whose burst structure is similar to that of GSM. Further results will be presented elsewhere for MSK, GMSK and OQAM modulations and applied to radiocommunications networks using these modulations such as the GSM or VAMOS network for example.

\section{REFERENCES}

[1] J. Benesty, J. Chen, and Y. Huang, "A widely linear distortionless filter for single-channel noise reduction", IEEE Signal Processing Letter, vol. 17, no. 5, pp. 469-472, May 2010.

[2] J. Benesty, J. Chen, and Y. Huang, "On widely linear Wiener and tradeoff filters for noise reduction", Speech Communications, doi: 10.1016/j8specom.2010.02.003, 2010.

[3] J. Capon, "High resolution frequency wave number spectrum analysis", Proc. IEEE, vol. 57, no. 8, pp. 1408-1418, Aug. 1969.

[4] J. Capon, R.J. Greenfield, and R.J. Kolker, "Multidimensional maximum likelihood processing of a large aperture seismic array", Proc. IEEE, vol. 55, no. 2, pp. 191-211, Feb. 1967.

[5] J.F. Cardoso and A. Souloumiac, "Blind beamforming for non-gaussian signals", IEE Proceedings-F, vol. 140, no. 6, pp 362-370, Dec. 1993.

[6] S. Chen, S. Tan, and L. Hanzo, "Adaptive beamforming for binary phase shift keying communication systems", Signal Processing, vol. 87, pp. 68-78, 2007.

[7] P. Chevalier, "Optimal time invariant and widely linear spatial filtering for radiocommunications", Proc. EUSIPCO, pp. 559-562, Triestre (Italy), Sept. 1996. 
[8] P. Chevalier and A. Blin, "Widely linear MVDR beamformers for the reception of an unknown signal corrupted by noncircular interferences", IEEE Trans. Signal Processing, vol. 55, no. 11, pp. 5323-5336, Nov 2007.

[9] P. Chevalier, J.P. Delmas, and A. Oukaci, "Optimal widely linear MVDR beamforming for noncircular signals," International Conference on Acoustics, Speech and Signal Processing, Taipei, Taiwan, April 2009.

[10] P. Chevalier, J.P. Delmas, and A. Oukaci, "Performance of Optimal Widely Linear MVDR beamforming for Noncircular Signals", Proc. EUSIPCO, Glasgow (Scotland), Aug. 2009.

[11] P. Chevalier and F. Pipon, "New Insights into optimal widely linear array receivers for the demodulation of BPSK, MSK and GMSK signals corrupted by noncircular interferences - Application to SAIC", IEEE Trans. Signal Processing, vol. 54, no. 3, pp. 870-883, March 2006.

[12] P. Chevalier, F. Pipon, and F. Delaveau, "Second order optimal array receivers for synchronization of BPSK, MSK and GMSK signals corrupted by noncircular interferences," Eurasip Journal on Advances in Signal Processing (JASP), Hindawi Publishing Corporation, vol. 2007, Article ID 45605, 2007.

[13] P. Chevalier and F. Dupuy, "Widely linear Alamouti receivers for the reception of real-valued signals corrupted by interferences The Alamouti-SAIC/MAIC concept", IEEE Trans. Signal Processing, vol. 59, no. 7, pp. 3339-3354, July 2011.

[14] J.P. Delmas, P. Chevalier, and S. Sallem, "Sensitivity of SAIC and MAIC concepts to residual frequency offsets", Proc. EUSIPCO, Aalborg (Denmark), Aug. 2010.

[15] A. Ferreol and P. Chevalier, "On the behavior of current second and higher order blind source separation methods for cyclostationary sources", IEEE Trans. Signal Processing, vol. 48, no. 6, pp. 1712-1725, June 2000. Errata vol. 50, no. 4, p. 990, April 2002.

[16] W.A. Gardner, "Signal interception, a unifying theorical framework for feature detection framework for feature detection", IEEE Trans. Communications vol. 36, no. 8, pp. 897-906, 1988

[17] L.J. Griffiths and C.W. Jim, "An alternative approach to linearly constrained adaptive beamforming", IEEE Trans. Antenna. Propagation, vol. 30, no. 1, pp. 27-34, Jan. 1982.

[18] P. Jallon, A. Chevreuil, P. Loubaton, and P. Chevalier, "Separation of convolutive mixtures of cyclostationary sources : a contrast function based approach", Proc. ICA 04, Grenade, Spain, September 2004.

[19] P. Jallon, A. Chevreuil, P. Loubaton, and P. Chevalier, "Separation of convolutive mixtures of linear modulated signals using constant modulus algorithm", International Conference on Acoustics, Speech and Signal Processing, Philadelphia, USA, 2005.

[20] C. Jutten and P. Comon, Handbook of Blind source separation, Independent Component Analysis and applications, Academic Press, 2010.

[21] M. Konrad and W. Gerstacker, "Interference robust transmission for the downlink of an OFDM-Based mobile communications system", EURASIP Journal on Wireless Communications and Networking, vol. 2008, Article ID 549371, 14 pages, doi: $10.1155 / 2008 / 549371$.

[22] K. Kuchi and V.K. Prabhu, "Performance evaluation for widely linear demodulation of PAM/QAM signals in the presence of Rayleigh fading and co-channel interference", IEEE Trans. Communications, vol. 57, no. 1, pp. 183-193, Jan. 2009.

[23] K. Kuchi and V.K. Prabhu, "Interference cancellation enhancement through generalized widely linear equalization in QAM systems", IEEE Trans. On Wireless Communications, vol 8, no. 4, pp. 1585-1590, April 2009.

[24] A. Lampe and M. Breiling, "Asymptotic analysis of widely linear MMSE multiuser detection- complex versus real modulation", Proc IEEE Info. Theory Workshop (ITW 2001), pp. 55-57, Sept 2001.

[25] D.P. Mandic and V.S.L. Goh, Complex valued nonlinear adaptive filters, noncircularity, widely linear and neural models", Wiley, 2009.

[26] R. Meyer, W.H. Gerstacker, and J.B Huber, "A single antenna interference cancellation algorithm for increased GSM capacity", IEEE Trans. Onn Wireless Com., vol 5, no. 7, pp. 1616-1621, July 2006.

[27] B. Picinbono, "On Circularity", IEEE Trans. Signal Processing vol 42, no. 12, pp. 3473-3482, Dec 1994. 
[28] B. Picinbono and P. Chevalier, "Widely linear estimation with complex data", IEEE Trans. Signal Processing, vol 43, no. 8, pp. 2030-2033, Aug. 1995.

[29] J.G. Proakis, Digital communications, McGraw-Hill, Third Edition, 1995.

[30] P. Schreier and L. Scharf, Statistical signal processing of complex-valued data, Cambridge University Press, 2010.

[31] H. Trigui and D.T.M. Slock, "Performance bounds for cochannel interference cancellation within the current GSM standard", Signal Proccessing, vol. 80, pp. 1335-1346, 2000.

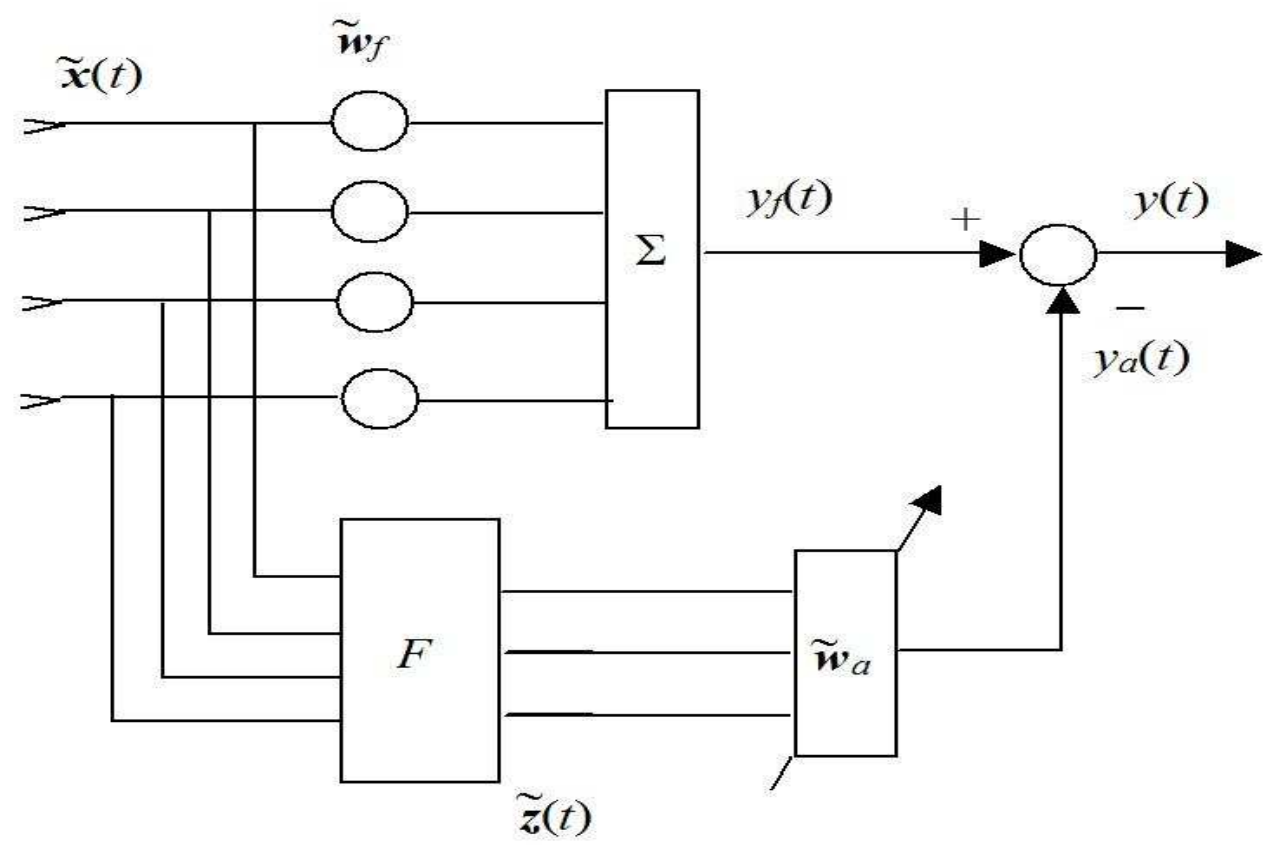

Figure 1. TI WL GSC structure of $\widetilde{\mathbf{w}}_{\mathrm{MVDR}_{2}}$. 


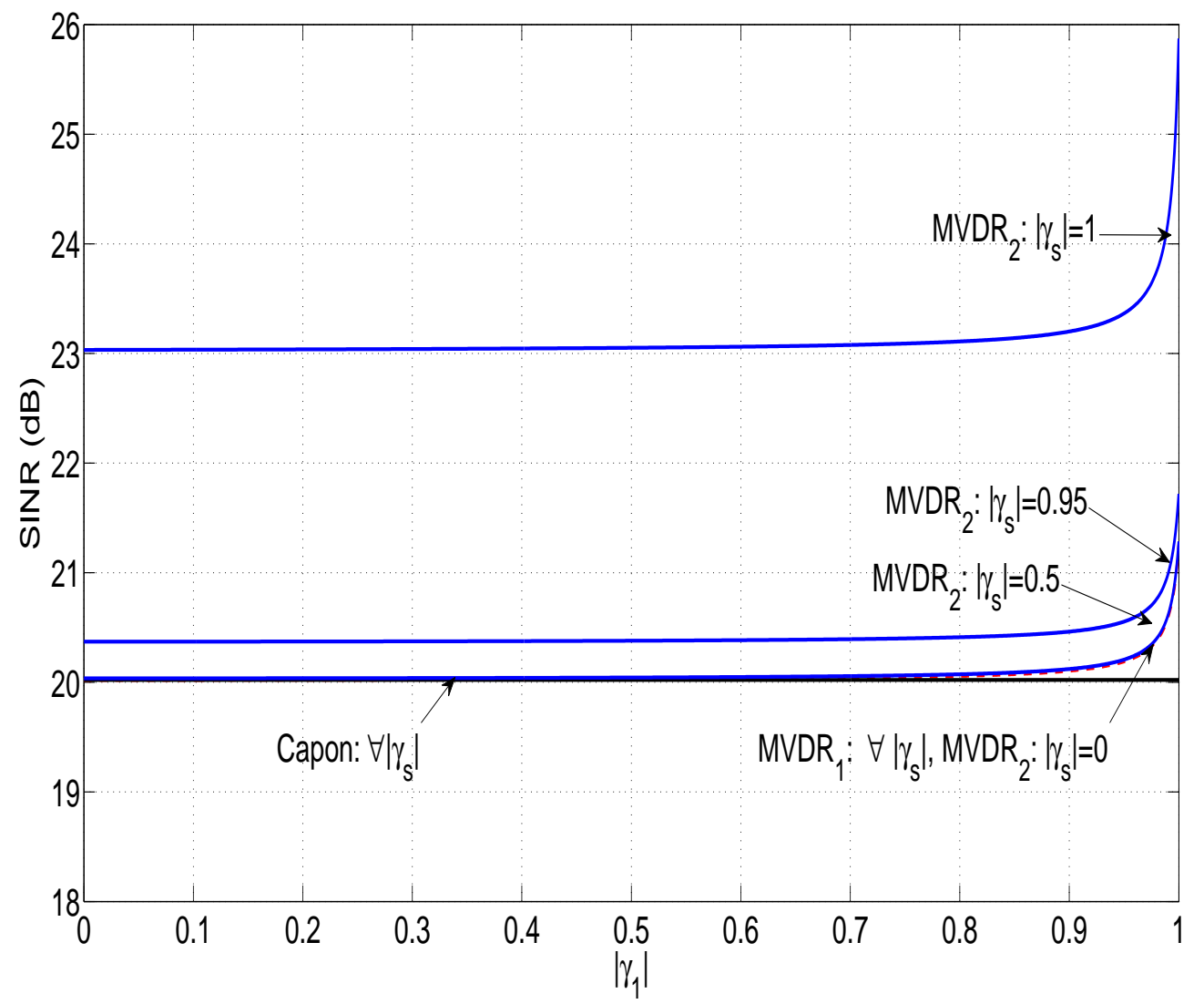

Figure 2. SINR $_{\mathrm{CAPON}}, \mathrm{SINR}_{\mathrm{MVDR}_{1}}$ and $\mathrm{SINR}_{\mathrm{MMSE}}=\mathrm{SINR}_{\mathrm{MVDR}_{2}}$ as a function of $\left|\gamma_{1}\right|$ for $N=2, P=1 \pi_{s} / \eta_{2}=20 \mathrm{~dB}$, $\pi_{1} / \eta_{2}=20 \mathrm{~dB}, \theta_{s}=0^{\circ}, \theta_{1}=30^{\circ}, \phi_{s}=0^{\circ}, \phi_{1}=60^{\circ},\left|\gamma_{s}\right|=0,0.5,0.95,1$. 


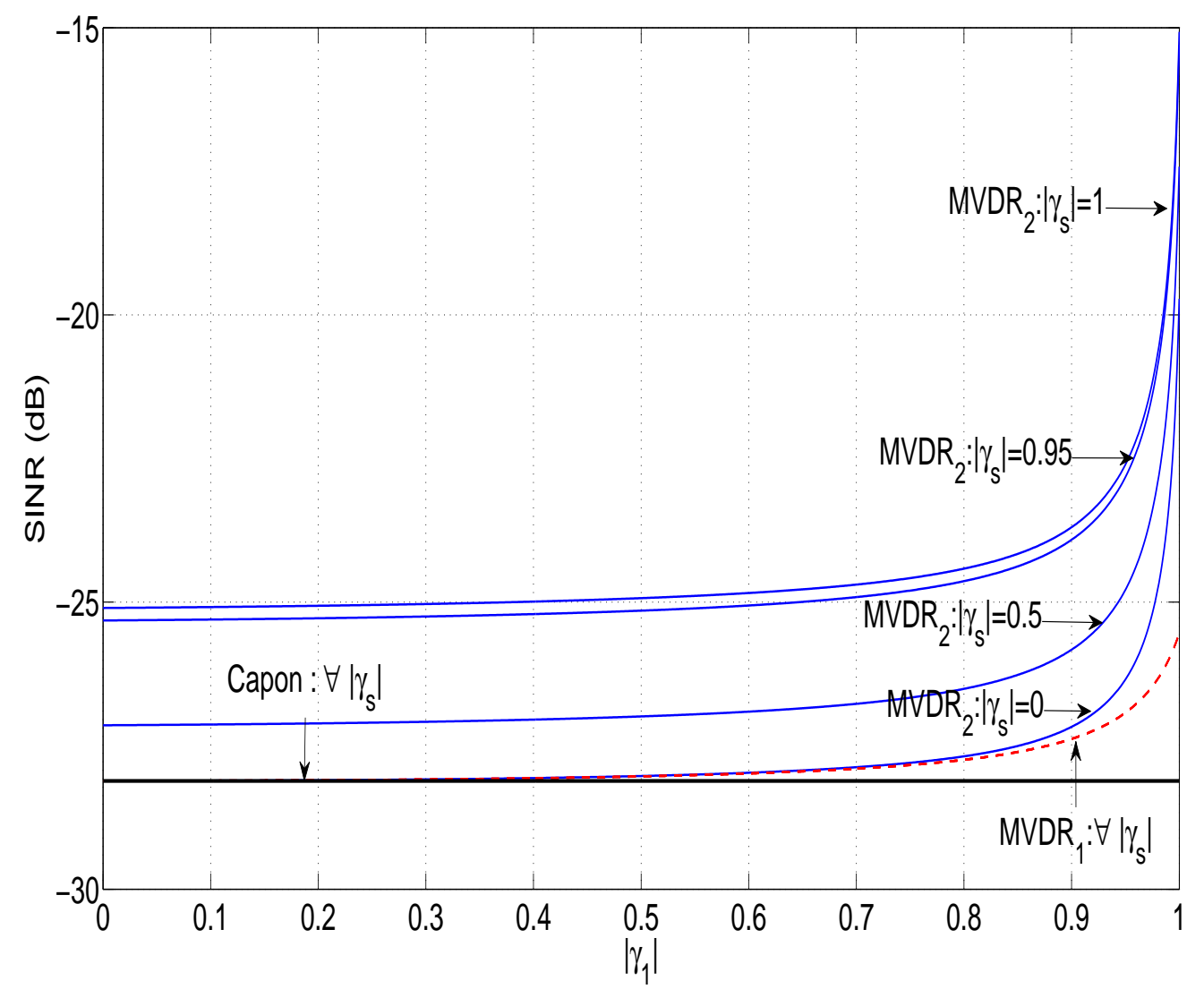

Figure 3. SINR $_{\mathrm{CAPON}}, \mathrm{SINR}_{\mathrm{MVDR}_{1}}$ and $\mathrm{SINR}_{\mathrm{MMSE}}=\mathrm{SINR}_{\mathrm{MVDR}_{2}}$ as a function of $\left|\gamma_{1}\right|$ for $N=2, P=1 \pi_{s} / \eta_{2}=-20 \mathrm{~dB}$, $\pi_{1} / \eta_{2}=20 \mathrm{~dB}, \theta_{s}=0^{\circ}, \theta_{1}=10^{\circ}, \phi_{s}=0^{\circ}, \phi_{1}=45^{\circ},\left|\gamma_{s}\right|=0,0.5,0.95,1$. 


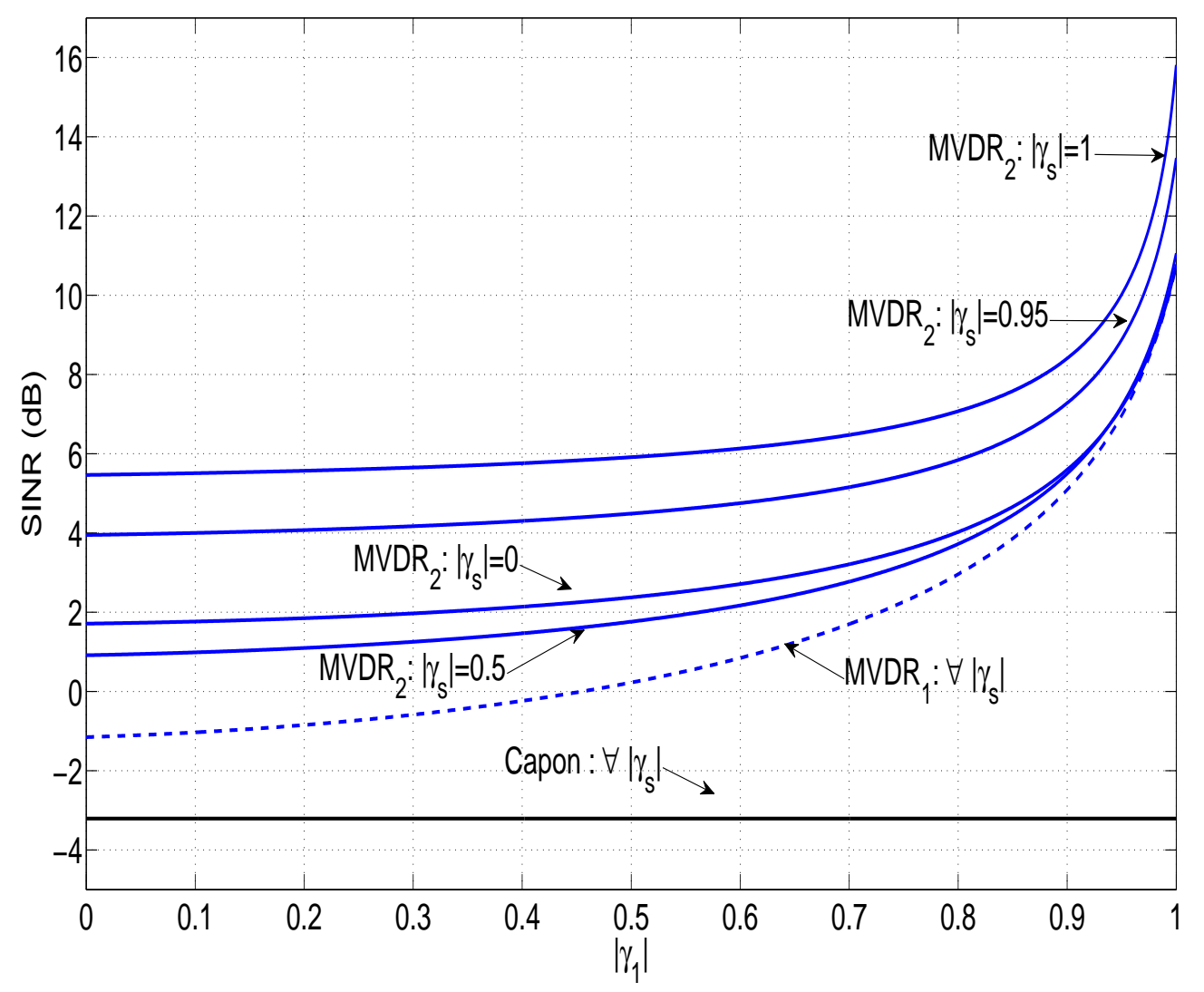

Figure 4. SINR $_{\mathrm{CAPON}}, \mathrm{SINR}_{\mathrm{MVDR}_{1}}$ and $\mathrm{SINR}_{\mathrm{MMSE}}=\mathrm{SINR}_{\mathrm{MVDR}_{2}}$ as a function of $\left|\gamma_{1}\right|$ for $N=2, P=2 \pi_{s} / \eta_{2}=10 \mathrm{~dB}$, $\pi_{1} / \eta_{2}=\pi_{2} / \eta_{2}=20 \mathrm{~dB}, \theta_{s}=0^{\circ}, \theta_{1}=30^{\circ}, \theta_{2}=60^{\circ}, \phi_{s}=0^{\circ}, \phi_{1}=60^{\circ}, \phi_{2}=80^{\circ},\left|\gamma_{2}\right|=1,\left|\gamma_{s}\right|=0,0.5,0.95,1$. 


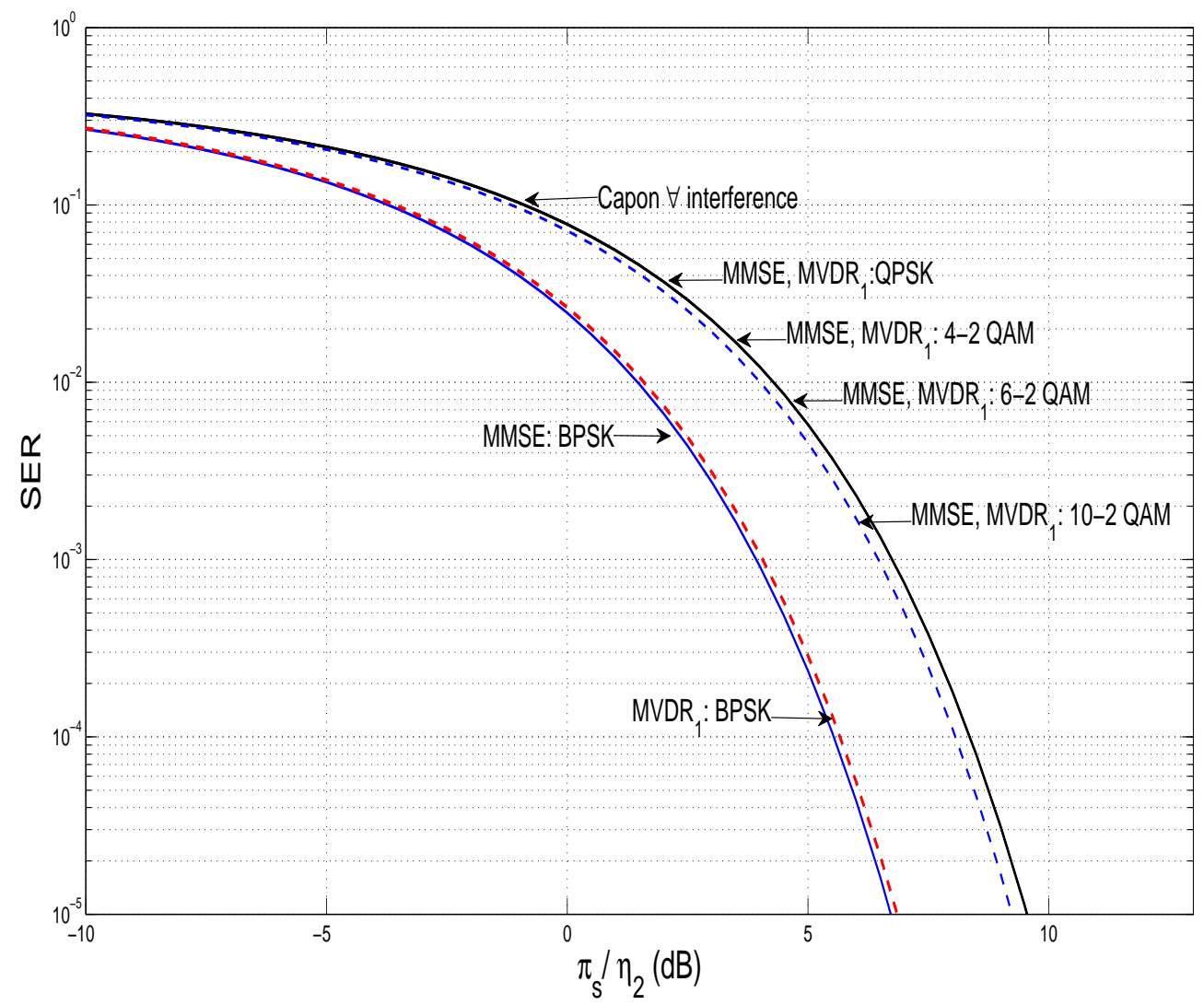

Figure 5. SER CAPON $_{\text {, }} \mathrm{SER}_{\mathrm{MVDR}_{1}}$ and $\mathrm{SER}_{\mathrm{MMSE}}=\mathrm{SER}_{\mathrm{MVDR}_{2}}$ as a function of $\pi_{s} / \eta_{2}$ for a BPSK SOI, $N=2, P=1$ $\pi_{1} / \eta_{2}=20 \mathrm{~dB}, \theta_{s}=0^{\circ}, \theta_{1}=30^{\circ}, \phi_{s}=0^{\circ}, \phi_{1}=60^{\circ},(U, V)=(2,2),(4,2),(6,2),(10,2),(2,1)$. 


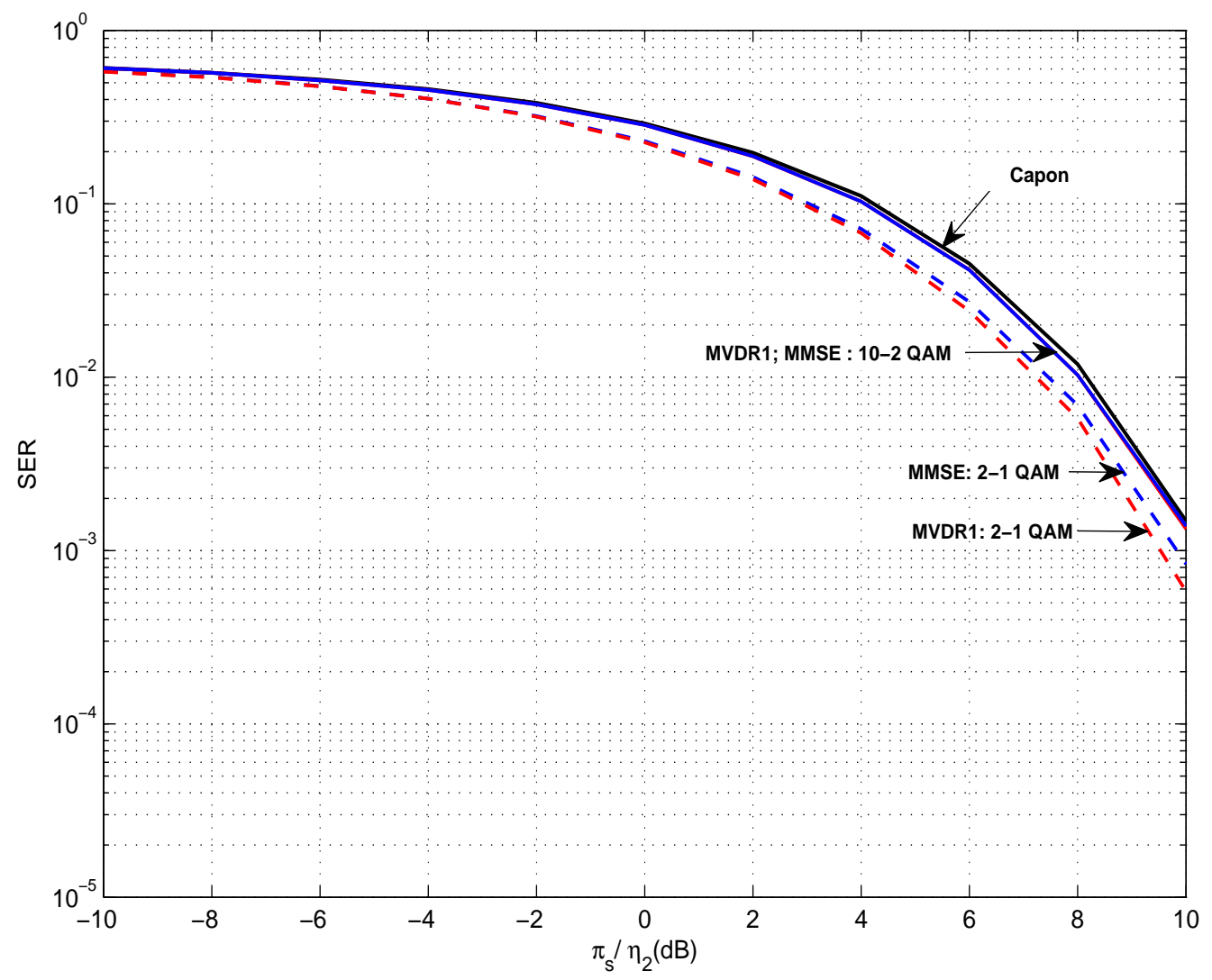

Figure 6. SER $\mathrm{CAPON}_{\mathrm{C}}, \mathrm{SER}_{\mathrm{MVDR}_{1}}$ and $\mathrm{SER}_{\mathrm{MMSE}}=\mathrm{SER}_{\mathrm{MVDR}_{2}}$ as a function of $\pi_{s} / \eta_{2}$ for a QPSK SOI, $N=2, P=1$ $\pi_{1} / \eta_{2}=20 \mathrm{~dB}, \theta_{s}=0^{\circ}, \theta_{1}=30^{\circ}, \phi_{s}=0^{\circ}, \phi_{1}=60^{\circ},(U, V)=(10,2),(2,1)$. 


\begin{tabular}{|c|c|c|c|c|c|c|}
\hline & $K=10$ & $K=20$ & $K=40$ & $K=80$ & $K=100$ & $K=1000$ \\
\hline$\widehat{M}=1$ & 0.395 & 0.026 & 0.071 & 0.081 & 0.162 & 0.033 \\
\hline$\widehat{M}=2$ & 0.420 & 0.139 & 0.114 & 0.150 & 0.226 & 0.032 \\
\hline
\end{tabular}

Table 1. $\left|\widehat{\gamma}_{o, \max }(K, \widehat{M})\right|$ as a function of $K$ and $\widehat{M}$ for scenario 1: (QPSK, QPSK), $N=2, M=2, T_{e}=T, \mu=0.3$, $\pi_{1} / \eta_{2}=\pi_{2} / \eta_{2}=10 \mathrm{~dB}, \theta_{1}=30^{\circ}, \theta_{2}=80^{\circ}, \phi_{1}=40^{\circ}, \phi_{2}=80^{\circ}$.

\begin{tabular}{|c|c|c|c|c|c|c|}
\hline & $K=10$ & $K=20$ & $K=40$ & $K=80$ & $K=100$ & $K=1000$ \\
\hline$\widehat{M}=1$ & 0.863 & 0.380 & 0.512 & 0.521 & 0.556 & 0.460 \\
\hline$\widehat{M}=2$ & 0.897 & 0.873 & 0.882 & 0.902 & 0.901 & 0.912 \\
\hline
\end{tabular}

Table 2. $\left|\widehat{\gamma}_{o, \max }(K, \widehat{M})\right|$ as a function of $K$ and $\widehat{M}$ for scenario 2: (BPSK, QPSK), $N=2, M=2, T_{e}=T, \mu=0.3$, $\pi_{1} / \eta_{2}=\pi_{2} / \eta_{2}=10 \mathrm{~dB}, \theta_{1}=30^{\circ}, \theta_{2}=80^{\circ}, \phi_{1}=40^{\circ}, \phi_{2}=80^{\circ}$.

\begin{tabular}{|c|c|c|c|c|c|c|}
\hline & $K=10$ & $K=20$ & $K=40$ & $K=80$ & $K=100$ & $K=1000$ \\
\hline$\widehat{M}=1$ & 0.209 & 0.088 & 0.437 & 0.113 & 0.057 & 0.152 \\
\hline$\widehat{M}=2$ & 0.979 & 0.939 & 0.894 & 0.936 & 0.939 & 0.908 \\
\hline
\end{tabular}

Table 3. $\left|\widehat{\gamma}_{o, \max }(K, \widehat{M})\right|$ as a function of $K$ and $\widehat{M}$ for scenario 3: (BPSK, BPSK), $N=2, M=2, T_{e}=T, \mu=0.3$, $\pi_{1} / \eta_{2}=\pi_{2} / \eta_{2}=10 \mathrm{~dB}, \theta_{1}=30^{\circ}, \theta_{2}=80^{\circ}, \phi_{1}=40^{\circ}, \phi_{2}=80^{\circ}$. 